\title{
Optimal and Nonlinear Dynamic Countermeasure under a Node-Level Model with Nonlinear Infection Rate
}

\author{
Xulong Zhang ${ }^{1}$ and Chenquan Gan ${ }^{1,2}$ \\ ${ }^{1}$ College of Computer Science, Chongqing University, Chongqing 400044, China \\ ${ }^{2}$ School of Communication and Information Engineering, Chongqing University of Posts and Telecommunications, \\ Chongqing 400065, China \\ Correspondence should be addressed to Chenquan Gan; gcq2010cqu@163.com
}

Received 20 January 2017; Accepted 15 May 2017; Published 12 June 2017

Academic Editor: Jose R. C. Piqueira

Copyright (C) 2017 Xulong Zhang and Chenquan Gan. This is an open access article distributed under the Creative Commons Attribution License, which permits unrestricted use, distribution, and reproduction in any medium, provided the original work is properly cited.

\begin{abstract}
This paper mainly addresses the issue of how to effectively inhibit viral spread by means of dynamic countermeasure. To this end, a controlled node-level model with nonlinear infection and countermeasure rates is established. On this basis, an optimal control problem capturing the dynamic countermeasure is proposed and analyzed. Specifically, the existence of an optimal dynamic countermeasure scheme and the corresponding optimality system are shown theoretically. Finally, some numerical examples are given to illustrate the main results, from which it is found that (1) the proposed optimal strategy can achieve a low level of infections at a low cost and (2) adjusting nonlinear infection and countermeasure rates and tradeoff factor can be conductive to the containment of virus propagation with less cost.
\end{abstract}

\section{Introduction}

In order to study the long-term behavior of computer virus and suppress viral spread macroscopically, a large number of dynamical models have been proposed in the past few decades (for the related references, see, e.g., [1-11]). From the perspective of the division scale of computers on networks, these models can be roughly divided into two categories: compartment-level models and node-level models.

Compartment-level models are those models that regard computers having the same state as an object to study. This work can be traced back to the 1980s. The first compartment-level model is proposed by Kephart and White [1], who followed the suggestions recommended by Cohen [12] and Murray [13]. Since then, multifarious propagation models have been developed (see, e.g., [14-22]). It is worth noticing that Zhu et al. [6] proposed the original compartment-level SICS (susceptible-infected-countermeasured-susceptible) model with linear static countermeasure based on the CMC (Countermeasure Competing) strategy presented by Chen and Carley [23]. However, compartment-level models ignore the effect of network eigenvalue on viral spread. Consequently, node-level models are considered.

Node-level models are those models that regard a single computer as an object to investigate. The first node-level model (i.e., SIS (susceptible-infected-susceptible) model) is proposed by Van Mieghem et al. [7]. Since then, Sahneh and Scoglio [8] presented the node-level SAIS (susceptible-alertinfected-susceptible) model, and Yang et al. $[9,10]$ considered the node-level SLBS (susceptible-latent-breaking-susceptible) and SIRS (susceptible-infected-recovered-susceptible) models, respectively. Very recently, Gan [11] established the node-level SIES (susceptible-infected-external-susceptible) model. Besides, for the other related work about this topic, one can refer to [24-28] and the references cited therein.

Inspired by the above-mentioned work and based on the compartment-level SICS model, this paper considers a controlled node-level SICS model. Different from the conventional node-level models, this paper mainly addresses the issue of how to effectively distribute dynamic countermeasure by optimal control strategy (for the related references of optimal models, see, e.g., [29-33]). An optimal control 


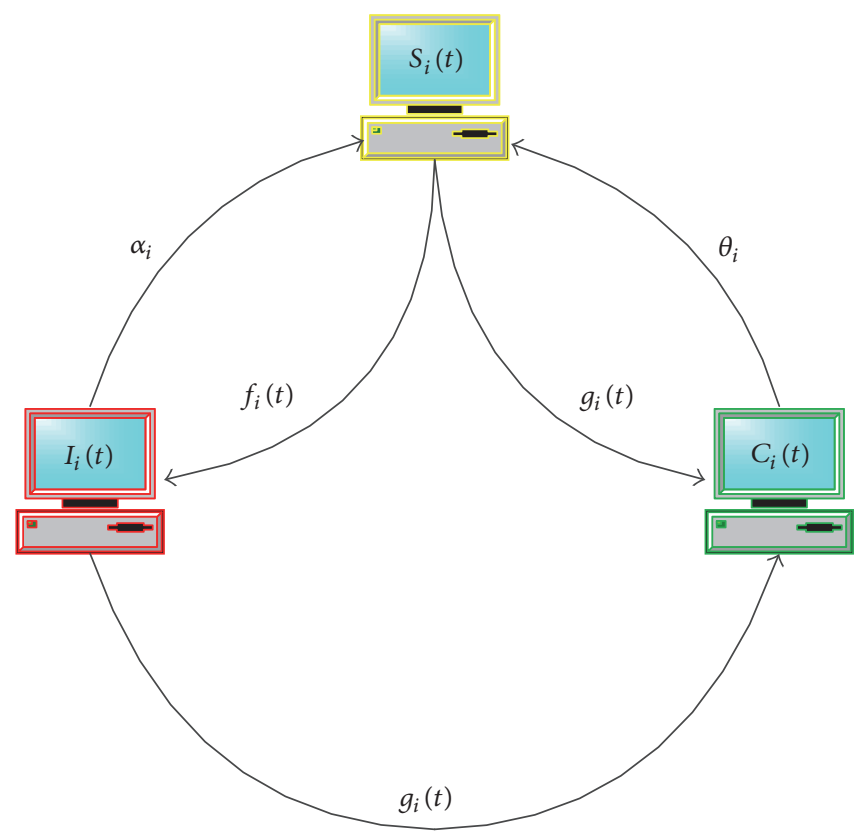

FIGURE 1: The transfer diagram of the controlled node-level SICS model.

problem is proposed and the existence of an optimal control is proved. The corresponding optimality system is also derived. Finally, some numerical examples are made, from which it can be seen that the proposed optimal strategy can achieve a low level of infections at a low cost.

The subsequent materials of this paper are organized as follows. Sections 2 and 3 formulate the controlled node-level model and analyze the optimal control problem, respectively. Numerical examples are provided in Section 4. Finally, Section 5 closes this work.

\section{The Controlled Node-Level Model}

In this paper, the propagation network of computer virus and countermeasure is represented by a graph $G=(V, E)$ with $N$ nodes labelled $1,2, \ldots, N$, where each node and edge stand for a computer and a network link, respectively. Thus, the graph $G$ can be described by its adjacency matrix $\mathbf{A}=$ $\left[a_{i j}\right]_{N \times N}$, where $a_{i i}=0$.

As was treated in the traditional SICS model [6], at any time all nodes in the graph $G$ are divided into three groups: $S$-nodes (susceptible nodes are uninfected but have no immunity), $I$-nodes (infected nodes), and $C$-nodes (countermeasured nodes are uninfected and have temporary immunity due to the presence of countermeasures). Let $S_{i}(t), I_{i}(t)$, and $C_{i}(t)$ denote the probability of node $i$ being susceptible, infected, and countermeasured at time $t$, respectively. Then the vector

$$
\left(S_{1}(t), \ldots, S_{N}(t), I_{1}(t), \ldots, I_{N}(t), C_{1}(t), \ldots, C_{N}(t)\right)^{T}
$$

probabilistically captures the state of the network at time $t$.
For convenience, two important functions, which will be used in the sequel, are defined as follows:

$$
f_{i}(t)=\sum_{j} \frac{a_{i j} \beta_{j} I_{j}(t)}{1+m_{1} I_{j}(t)}, \quad m_{1} \geq 0, \beta_{j}>0 .
$$

Clearly, $f_{i}(t) \leq \sum_{j} a_{i j} \beta_{j} I_{j}(t)$.

$$
g_{i}(t)=\sum_{j} \frac{a_{i j} \gamma_{j}(t) C_{j}(t)}{1+m_{2} C_{j}(t)}, \quad m_{2} \geq 0,
$$

where $\gamma_{j}(t) \in L^{2}[0, T]$ is a controllable rate, $\underline{\gamma} \leq \gamma_{j}(t) \leq \bar{\gamma}$, $0 \leq t \leq T ; \gamma$ and $\bar{\gamma}$ are positive constants, $0<\bar{\gamma}<\bar{\gamma}<1$.

Now, a set of probabilistic assumptions on the state transition of node $i$ are made (see also Figure 1).

(A1) An $S$-node $i$ becomes infected at rate $f_{i}(t)$.

(A2) An $S$ - or $I$-node $i$ becomes countermeasured at rate $g_{i}(t)$.

(A3) An $I$-node $i$ becomes susceptible at a constant rate $\alpha_{i}>0$.

(A4) A $C$-node $i$ loses immunity at constant rate $\theta_{i}>0$.

Let $\Delta t$ be a very small time interval and $o(\Delta t)$ be a higherorder infinitesimal. Assumptions (A1)-(A4) imply that the probabilities of state transition of node $i$ satisfy the following relations:

$$
\begin{aligned}
& \operatorname{Pr}(i \text { is infected at time } t \\
& +\Delta t \mid i \text { is susceptible at time } t)=f_{i}(t) \Delta t \\
& +o(\Delta t),
\end{aligned}
$$


$\operatorname{Pr}(i$ is countermeasured at time $t$

$+\Delta t \mid i$ is susceptible at time $t)=g_{i}(t) \Delta t$

$+o(\Delta t)$,

$\operatorname{Pr}(i$ is countermeasured at time $t$

$+\Delta t \mid i$ is infected at time $t)=g_{i}(t) \Delta t+o(\Delta t)$,

$\operatorname{Pr}(i$ is susceptible at time $t$

$+\Delta t \mid i$ is infected at time $t)=\alpha_{i} \Delta t+o(\Delta t)$,

$\operatorname{Pr}(i$ is susceptible at time $t$

$+\Delta t \mid i$ is countermeasured at time $t)=\theta_{i} \Delta t$

$+o(\Delta t)$.

(4)

Invoking the total probability formulas and letting $\Delta t \rightarrow$ 0 , the controlled node-level model (i.e., controlled node-level SICS model) can be derived.

$$
\begin{aligned}
& \frac{d S_{i}(t)}{d t}=\alpha_{i} I_{i}(t)+\theta_{i} C_{i}(t)-f_{i}(t) S_{i}(t)-g_{i}(t) S_{i}(t), \\
& \frac{d I_{i}(t)}{d t}=-\alpha_{i} I_{i}(t)+f_{i}(t) S_{i}(t)-g_{i}(t) I_{i}(t), \\
& \frac{d C_{i}(t)}{d t}=-\theta_{i} C_{i}(t)+g_{i}(t)\left(S_{i}(t)+I_{i}(t)\right), \\
& \quad 0 \leq t \leq T, i=1,2, \ldots, N,
\end{aligned}
$$

with initial condition

$$
\begin{aligned}
& \left(S_{1}(0), \ldots, S_{N}(0), I_{1}(0), \ldots, I_{N}(0), C_{1}(0), \ldots, C_{N}(0)\right)^{T} \\
& \quad \in \widetilde{\Omega},
\end{aligned}
$$

where

$$
\begin{aligned}
\widetilde{\Omega} & =\left\{\left(S_{1}, \ldots, S_{N}, I_{1}, \ldots, I_{N}, C_{1}, \ldots, C_{N}\right)^{T} \in \mathbb{R}_{+}^{3 N} \mid S_{i}\right. \\
& \left.+I_{i}+C_{i}=1, i=1,2, \ldots, N\right\} .
\end{aligned}
$$

The admissible control set is

$$
U=\left\{\mathbf{u}(\cdot) \in\left(L^{2}[0, T]\right)^{N} \mid \underline{\gamma} \leq \gamma_{i}(\cdot) \leq \bar{\gamma}, 1 \leq i \leq N\right\},
$$

where $\mathbf{u}(\cdot)=\left(\gamma_{1}(\cdot), \ldots, \gamma_{N}(\cdot)\right)^{T}$.

\section{The Optimal Control Problem}

As $S_{i}(t)+I_{i}(t)+C_{i}(t) \equiv 1,1 \leq i \leq N$, system (5) can be reduced to the following system:

$$
\begin{aligned}
\frac{d I_{i}(t)}{d t}= & -\alpha_{i} I_{i}(t)+f_{i}(t)\left(1-I_{i}(t)-C_{i}(t)\right) \\
& -g_{i}(t) I_{i}(t), \\
\frac{d C_{i}(t)}{d t}= & -\theta_{i} C_{i}(t)+g_{i}(t)\left(1-C_{i}(t)\right), \\
& 0 \leq t \leq T, i=1,2, \ldots, N, N
\end{aligned}
$$

with initial condition

$$
\left(I_{1}(0), \ldots, I_{N}(0), C_{1}(0), \ldots, C_{N}(0)\right)^{T} \in \Omega,
$$

where

$$
\begin{aligned}
\Omega & =\left\{\left(I_{1}, \ldots, I_{N}, C_{1}, \ldots, C_{N}\right)^{T} \in \mathbb{R}_{+}^{2 N} \mid I_{i}+C_{i} \leq 1, i\right. \\
& =1,2, \ldots, N\} .
\end{aligned}
$$

Then system (9) can be written in matrix notation as

$$
\frac{d \mathbf{x}(t)}{d t}=\mathbf{f}(\mathbf{x}(t), \mathbf{u}(t)), \quad 0 \leq t \leq T,
$$

with initial condition $\mathbf{x}(0) \in \Omega$.

Now, the objective is to find a control variable $\mathbf{u}(\cdot) \in U$ so as to minimize both the prevalence of infected computers and the total budget for dynamic countermeasure during the time period $[0, T]$. That is, the following optimal control problem needs to be solved:

$$
\underset{\mathbf{u} \in U}{\operatorname{Minimize}} J(\mathbf{u})=\int_{0}^{T} L(\mathbf{x}(t), \mathbf{u}(t)) d t
$$

subject to system (12), where

$$
L(\mathbf{x}, \mathbf{u})=\sum_{i}\left(I_{i}(t)+\frac{1}{2} \varepsilon_{i} \gamma_{i}^{2}(t)\right), \quad \varepsilon_{i}>0,
$$

is the Lagrangian and $\varepsilon=\left(\varepsilon_{1}, \ldots, \varepsilon_{N}\right)^{T}$ is a tradeoff factor based on the control effect and control cost of dynamic countermeasure.

3.1. Existence of an Optimal Control. First, a lemma, which plays a critical role afterwards, is introduced.

Lemma 1 (see $[34,35])$. We have an optimal control problem

$$
\underset{\mathbf{u} \in U}{\operatorname{Minimize}} J(\mathbf{u})=\int_{0}^{T} L(\mathbf{x}(t), \mathbf{u}(t)) d t
$$

subject to

$$
\frac{d \mathbf{x}(t)}{d t}=\mathbf{f}(\mathbf{x}(t), \mathbf{u}(t)), \quad 0 \leq t \leq T,
$$

with $\mathbf{x}(0) \in \Omega$, where $\Omega$ is positively invariant for system (15). The problem has an optimal control if the following six conditions hold simultaneously.

(C1) There is $\mathbf{u} \in U$ such that system (15) is solvable.

(C2) $U$ is convex.

(C3) $U$ is closed.

(C4) $\mathbf{f}(\mathbf{x}, \mathbf{u})$ is bounded by a linear function in $\mathbf{x}$.

(C5) $L(\mathbf{x}, \mathbf{u})$ is convex on $U$.

(C6) $L(\mathbf{x}, \mathbf{u}) \geq c_{1}\|\mathbf{u}\|_{2}^{\rho}+c_{2}$ for some $\rho>1, c_{1}>0$, and $c_{2}$.

In order to prove the existence of an optimal control, six lemmas, one for each condition in Lemma 1, should be proved. 
Lemma 2. There is $\mathbf{u} \in U$ such that system (9) or (12) is solvable.

Proof. Substituting $\mathbf{u} \equiv \overline{\mathbf{u}}:=(\underbrace{\bar{\gamma}, \ldots, \bar{\gamma}}_{N})^{T}$ into system (12), one can get the uncontrolled system:

$$
\frac{d \mathbf{x}(t)}{d t}=\mathbf{f}(\mathbf{x}(t), \overline{\mathbf{u}})
$$

with $\mathbf{x}(0) \in \Omega$. Then the function $\mathbf{f}(\mathbf{x}, \overline{\mathbf{u}})$ is continuously differentiable, and $\Omega$ is positively invariant for the system. Hence, the claimed result follows from the Continuation Theorem for differential equations [36].

Lemma 3. The admissible set $U$ is convex.

Proof. Let

$$
\begin{aligned}
& \mathbf{u}^{(1)}=\left(\gamma_{1}^{(1)}, \ldots, \gamma_{N}^{(1)}\right) \in U, \\
& \mathbf{u}^{(2)}=\left(\gamma_{1}^{(2)}, \ldots, \gamma_{N}^{(2)}\right) \in U, \\
& \qquad<<<1 .
\end{aligned}
$$

As $\left(L^{2}[0, T]\right)^{N}$ is a real vector space, one can obtain

$$
(1-\xi) \mathbf{u}^{(1)}+\xi \mathbf{u}^{(2)} \in\left(L^{2}[0, T]\right)^{N}
$$

Then, the convexity of $U$ follows by the observation that

$$
\underline{\gamma} \leq(1-\xi) \gamma_{i}^{(1)}+\xi \gamma_{i}^{(2)} \leq \bar{\gamma}, \quad 1 \leq i \leq N .
$$

Hence, the claimed result follows.

Lemma 4. The admissible set $U$ is closed.

Proof. Let $\mathbf{u}=\left(\gamma_{1}, \ldots, \gamma_{N}\right)^{T}$ be a limit point of $U$ and

$$
\mathbf{u}^{(n)}=\left(\gamma_{1}^{(n)}, \ldots, \gamma_{N}^{(n)}\right)^{T}, \quad n=1,2, \ldots
$$

be a sequence of points in $U$ such that

$$
\left\|\mathbf{u}^{(n)}-\mathbf{u}\right\|_{2}:=\left[\int_{0}^{T}\left|\mathbf{u}^{(n)}(t)-\mathbf{u}(t)\right|^{2} d t\right]^{1 / 2}<\frac{1}{n} .
$$

From the completeness of $\left(L^{2}[0, T]\right)^{N}$, one can get

$$
\lim _{n \rightarrow \infty} \mathbf{u}^{(n)}=\mathbf{u} \in\left(L^{2}[0, T]\right)^{N} .
$$

Hence, the closeness of $U$ follows from the observation that

$$
\underline{\gamma} \leq \gamma_{i}=\lim _{n \rightarrow \infty} \gamma_{i}^{(n)} \leq \bar{\gamma}, \quad 1 \leq i \leq N .
$$

Lemma 5. $\mathbf{f}(\mathbf{x}, \mathbf{u})$ is bounded by a linear function in $\mathbf{x}$.
Proof. Note that, for system (9) and for $i=1,2, \ldots, N$,

$$
\begin{aligned}
-\frac{\bar{\gamma} N^{2}}{4}-\alpha_{i} I_{i} & \leq \frac{d I_{i}}{d t} \leq-\alpha_{i} I_{i}+\sum_{j} a_{i j} \beta_{j} I_{j}, \\
-\theta_{i} C_{i} & \leq \frac{d C_{i}}{d t} \leq-\theta_{i} C_{i}+\bar{\gamma} \sum_{j} a_{i j} C_{j} .
\end{aligned}
$$

Thus, the claimed result follows.

Lemma 6. $L(\mathbf{x}, \mathbf{u})$ is convex on $U$.

Proof. Note that the Hessian matrix of $L(\mathbf{x}, \mathbf{u})$ with respect to $\mathbf{u} \in U$ is as follows:

$\mathbf{H}_{\mathbf{u}}(L)$

$$
=\left[\begin{array}{ccccc}
\frac{\partial^{2} L}{\partial \gamma_{1}^{2}} & \frac{\partial^{2} L}{\partial \gamma_{1} \partial \gamma_{2}} & \cdots & \frac{\partial^{2} L}{\partial \gamma_{1} \partial \gamma_{N-1}} & \frac{\partial^{2} L}{\partial \gamma_{1} \partial \gamma_{N}} \\
\frac{\partial^{2} L}{\partial \gamma_{2} \partial \gamma_{1}} & \frac{\partial^{2} L}{\partial \gamma_{2}^{2}} & \cdots & \frac{\partial^{2} L}{\partial \gamma_{2} \partial \gamma_{N-1}} & \frac{\partial^{2} L}{\partial \gamma_{2} \partial \gamma_{N}} \\
\vdots & \vdots & \ddots & \vdots & \vdots \\
\frac{\partial^{2} L}{\partial \gamma_{N-1} \partial \gamma_{1}} & \frac{\partial^{2} L}{\partial \gamma_{N-1} \partial \gamma_{2}} & \cdots & \frac{\partial^{2} L}{\partial \gamma_{N-1}^{2}} & \frac{\partial^{2} L}{\partial \gamma_{N-1} \partial \gamma_{N}} \\
\frac{\partial^{2} L}{\partial \gamma_{N} \partial \gamma_{1}} & \frac{\partial^{2} L}{\partial \gamma_{N} \partial \gamma_{2}} & \cdots & \frac{\partial^{2} L}{\partial \gamma_{N} \partial \gamma_{N-1}} & \frac{\partial^{2} L}{\partial \gamma_{N}^{2}}
\end{array}\right]
$$

$$
=\left[\begin{array}{ccccc}
\varepsilon_{1} & 0 & \cdots & 0 & 0 \\
0 & \varepsilon_{2} & \cdots & 0 & 0 \\
\vdots & \vdots & \ddots & \vdots & \vdots \\
0 & 0 & \cdots & \varepsilon_{N-1} & 0 \\
0 & 0 & \cdots & 0 & \varepsilon_{N}
\end{array}\right] .
$$

For any $t \in[0, T], \mathbf{H}_{\mathbf{u}}(L)$ is real symmetric and its eigenvalues are all positive. Then, $\mathbf{H}_{\mathbf{u}}(L)$ is positive definite. Hence, the convexity of $L(\mathbf{x}, \mathbf{u})$ follows by the result in [37].

Lemma 7. $L(\mathbf{x}, \mathbf{u}) \geq c_{1}\|\mathbf{u}\|_{2}^{\rho}+c_{2}$ for some $\rho>1, c_{1}>0$, and $c_{2}$.

Proof. Let $\rho=2, c_{1}=\min _{i}\left\{\varepsilon_{i}\right\} / 2$, and $c_{2}=0$. Then, $L(\mathbf{x}, \mathbf{u}) \geq$ $\left(\min _{i}\left\{\varepsilon_{i}\right\} / 2\right) \times\|\mathbf{u}\|_{2}^{2}$. Thus, the proof is complete.

Now, it is time to examine the main result of this subsection.

Theorem 8. The optimal control problem (P) has a solution.

Proof. Lemmas 2-7 show that the six conditions in Lemma 1 are all met. Hence, the proof is complete.

3.2. The Optimality System. In this subsection, a necessary condition for an optimal control of problem (P) is drawn.

Theorem 9. Suppose $\mathbf{u}^{*}(\cdot)$ is an optimal control for problem (P) and $\left(I^{*}(\cdot), C^{*}(\cdot)\right)^{T}$ is the solution to system (9) with 
$\mathbf{u}(\cdot)=\mathbf{u}^{*}(\cdot)$. Then, there exist functions $\lambda_{1 i}^{*}(t)$ and $\lambda_{2 i}^{*}(t)$, $0 \leq t \leq T, 1 \leq i \leq N$, such that

$$
\begin{aligned}
& \frac{d \lambda_{1 i}^{*}(t)}{d t}=-1+\lambda_{1 i}^{*}(t)\left[\alpha_{i}+f_{i}^{*}(t)+g_{i}^{*}(t)\right] \\
& -\frac{\beta_{i}}{\left(1+m_{1} I_{i}^{*}(t)\right)^{2}} \\
& \cdot \sum_{j} a_{i j} \lambda_{1 j}^{*}(t)\left(1-I_{j}^{*}(t)-C_{j}^{*}(t)\right), \\
& \frac{d \lambda_{2 i}^{*}(t)}{d t}=\lambda_{1 i}^{*}(t) f_{i}^{*}(t)+\lambda_{2 i}^{*}(t)\left[\theta_{i}+g_{i}^{*}(t)\right]
\end{aligned}
$$

$$
\begin{aligned}
& +\frac{\gamma_{i}^{*}(t)}{\left(1+m_{2} C_{i}^{*}(t)\right)^{2}} \\
& \cdot \sum_{j} a_{i j}\left[I_{j}^{*}(t) \lambda_{1 j}^{*}(t)-\left(1-C_{j}^{*}(t)\right) \lambda_{2 j}^{*}(t)\right], \\
& \quad 0 \leq t \leq T, i=1,2, \ldots, N,
\end{aligned}
$$

with transversality conditions

$$
\lambda_{1 i}^{*}(T)=\lambda_{2 i}^{*}(T)=0, \quad i=1,2, \ldots, N .
$$

Furthermore, one can get

$$
\gamma_{i}^{*}(t)=\max \left\{\min \left\{\frac{C_{i}^{*}(t)}{\varepsilon_{i}\left(1+m_{2} C_{i}^{*}(t)\right)} \sum_{j} a_{i j}\left[I_{j}^{*}(t) \lambda_{1 j}^{*}(t)-\left(1-C_{j}^{*}(t)\right) \lambda_{2 j}^{*}(t)\right], \bar{\gamma}\right\}, \underline{\gamma}\right\},
$$

$0 \leq t \leq T, i=1,2, \ldots, N$.

Proof. The corresponding Hamiltonian is

$$
\begin{aligned}
H(\mathbf{I}, \mathbf{C}, \lambda, \mathbf{u})= & \sum_{i}\left(I_{i}+\frac{1}{2} \varepsilon_{i} \gamma_{i}^{2}\right)+\sum_{i} \lambda_{1 i} \frac{d I_{i}}{d t} \\
& +\sum_{i} \lambda_{2 i} \frac{d C_{i}}{d t},
\end{aligned}
$$

where $\lambda_{1 i}, \lambda_{2 i}$ are undetermined, $\lambda=\left(\lambda_{11}, \ldots, \lambda_{1 N}, \lambda_{21}, \ldots\right.$, $\left.\lambda_{2 N}\right)^{T}$.

According to the Pontryagin Minimum Principle [35], there exist functions $\lambda_{1 i}^{*}(t)$ and $\lambda_{2 i}^{*}(t), 0 \leq t \leq T, 1 \leq i \leq N$, such that

$$
\begin{aligned}
& \frac{d \lambda_{1 i}^{*}(t)}{d t}=-\frac{\partial H\left(\mathbf{I}^{*}(t), \mathbf{C}^{*}(t), \lambda^{*}(t), \mathbf{u}^{*}(t)\right)}{\partial I_{i}}, \\
& \frac{d \lambda_{2 i}^{*}(t)}{d t}=-\frac{\partial H\left(\mathbf{I}^{*}(t), \mathbf{C}^{*}(t), \lambda^{*}(t), \mathbf{u}^{*}(t)\right)}{\partial C_{i}}, \\
& 0 \leq t \leq T, i=1,2, \ldots, N .
\end{aligned}
$$

Thus, system (26) follows by direct calculations. As the terminal cost is unspecified and the final state is free, the transversality conditions hold. By using the optimality condition

$$
H\left(\mathbf{I}^{*}, \mathbf{C}^{*}, \lambda^{*}, \mathbf{u}^{*}\right)=\min _{\mathbf{u} \in U} H\left(\mathbf{I}^{*}, \mathbf{C}^{*}, \lambda^{*}, \mathbf{u}\right),
$$

one can obtain that, for $0 \leq t \leq T$ and for $1 \leq i \leq N$, either

$$
\begin{aligned}
& \frac{\partial H\left(\mathbf{I}^{*}(t), \mathbf{C}^{*}(t), \lambda^{*}(t), \mathbf{u}^{*}(t)\right)}{\partial \gamma_{i}}=\varepsilon_{i} \gamma_{i}^{*}(t) \\
& -\frac{C_{i}^{*}(t)}{1+m_{2} C_{i}^{*}(t)} \\
& \cdot \sum_{j} a_{i j}\left[I_{j}^{*}(t) \lambda_{1 j}^{*}(t)-\left(1-C_{j}^{*}(t)\right) \lambda_{2 j}^{*}(t)\right]=0
\end{aligned}
$$

or $\gamma_{i}^{*}(t)=\underline{\gamma}$ or $\gamma_{i}^{*}(t)=\bar{\gamma}$. Hence, the proof is complete.

By combining the above discussions, one can get the optimality system for problem (P) as follows:

$$
\begin{aligned}
& \frac{d I_{i}(t)}{d t}=-\alpha_{i} I_{i}(t)+f_{i}(t)\left(1-I_{i}(t)-C_{i}(t)\right)-g_{i}(t) I_{i}(t), \\
& \frac{d C_{i}(t)}{d t}=-\theta_{i} C_{i}(t)+g_{i}(t)\left(1-C_{i}(t)\right), \\
& \frac{d \lambda_{1 i}(t)}{d t}=-1+\lambda_{1 i}(t)\left[\alpha_{i}+f_{i}(t)+g_{i}(t)\right]-\frac{\beta_{i}}{\left(1+m_{1} I_{i}(t)\right)^{2}} \sum_{j} a_{i j} \lambda_{1 j}(t)\left(1-I_{j}(t)-C_{j}(t)\right), \\
& \frac{d \lambda_{2 i}(t)}{d t}=\lambda_{1 i}(t) f_{i}(t)+\lambda_{2 i}(t)\left[\theta_{i}+g_{i}(t)\right]+\frac{\gamma_{i}(t)}{\left(1+m_{2} C_{i}(t)\right)^{2}} \sum_{j} a_{i j}\left[I_{j}(t) \lambda_{1 j}(t)-\left(1-C_{j}(t)\right) \lambda_{2 j}(t)\right],
\end{aligned}
$$




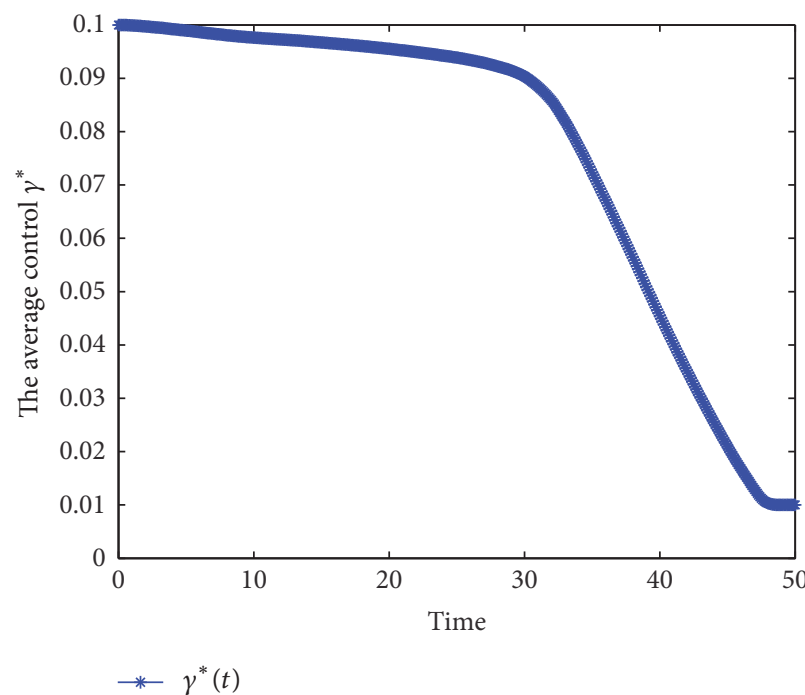

(a) $\gamma^{*}(t)$
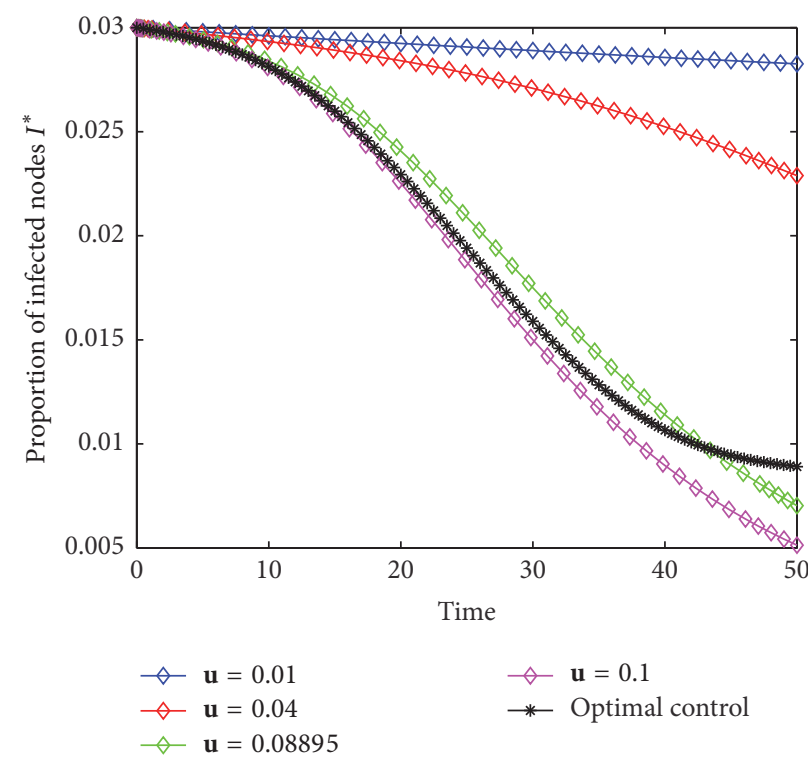

(b) $I^{*}(t)$

FIgURE 2: $\gamma^{*}(t)$ and $I^{*}(t)$ under different control strategies with $m_{1}=m_{2}=2$ for Example 1 .

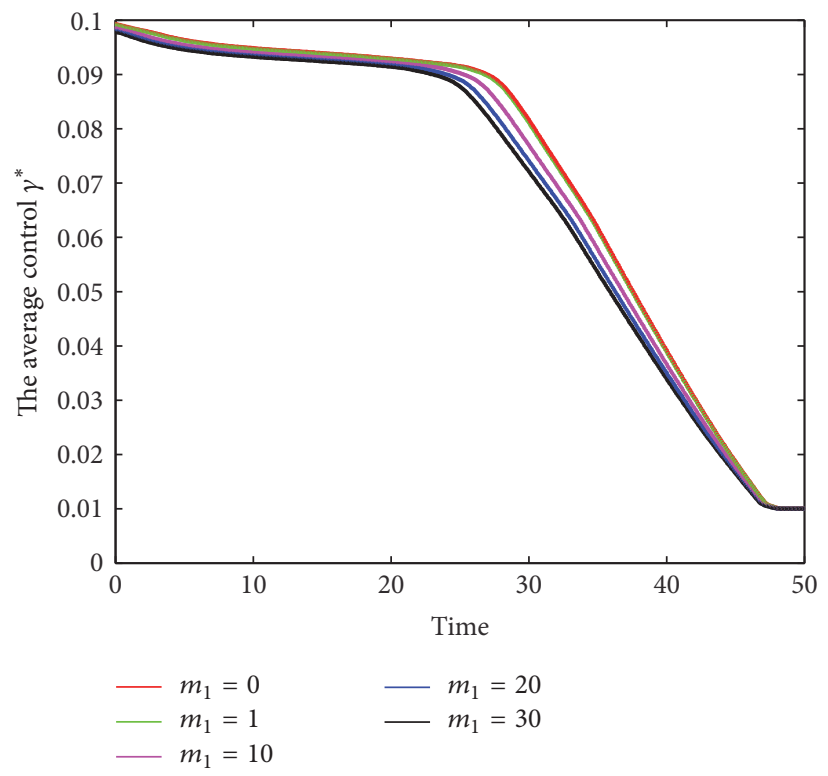

(a) $m_{2}=5$

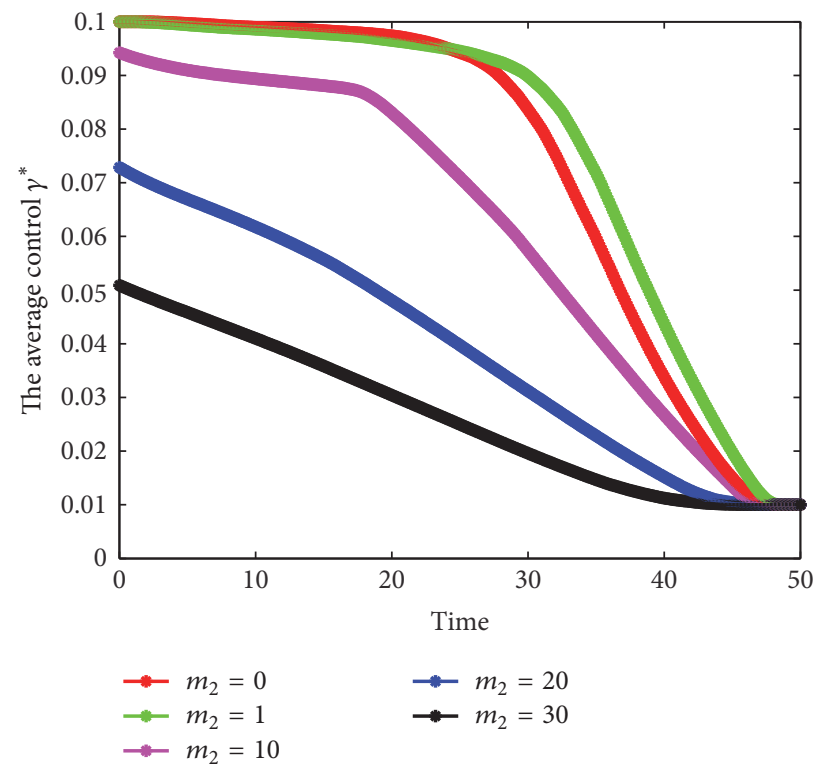

(b) $m_{1}=5$

FIgURE 3: $\gamma^{*}(t)$ for different $m_{1}$ and $m_{2}$ for Example 1 .

$$
\gamma_{i}(t)=\max \left\{\min \left\{\frac{C_{i}(t)}{\varepsilon_{i}\left(1+m_{2} C_{i}(t)\right)} \sum_{j} a_{i j}\left[I_{j}(t) \lambda_{1 j}(t)-\left(1-C_{j}(t)\right) \lambda_{2 j}(t)\right], \bar{\gamma}\right\}, \underline{\gamma}\right\},
$$

$0 \leq t \leq T, i=1,2, \ldots, N$

with $(\mathbf{I}(0), \mathbf{C}(0))^{T} \in \Omega$ and $\lambda(T)=0$. 


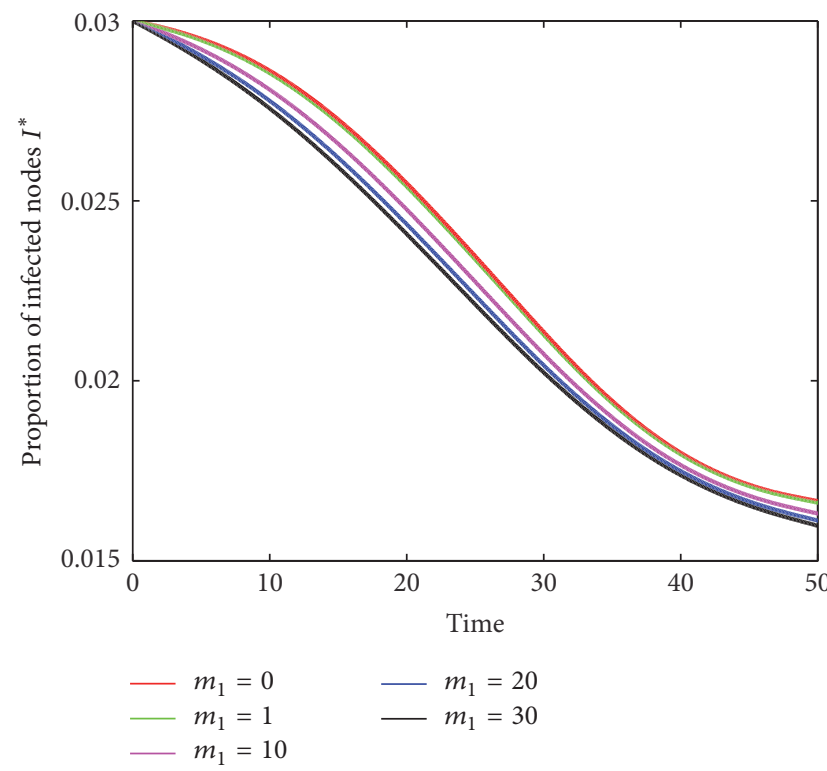

(a) $m_{2}=5$

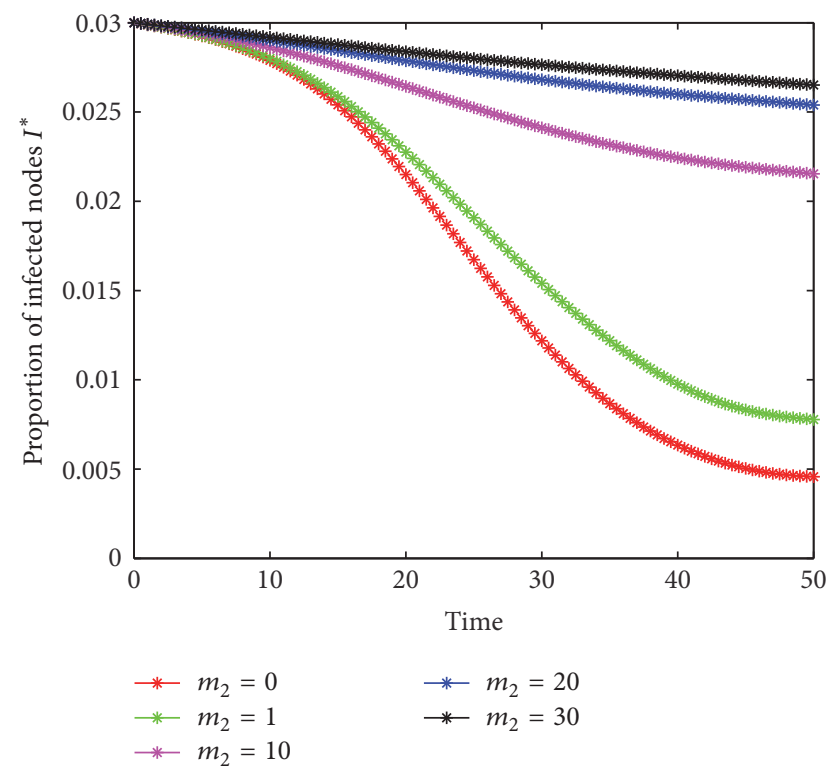

(b) $m_{1}=5$

FIgURE 4: $I^{*}(t)$ for different $m_{1}$ and $m_{2}$ for Example 1.

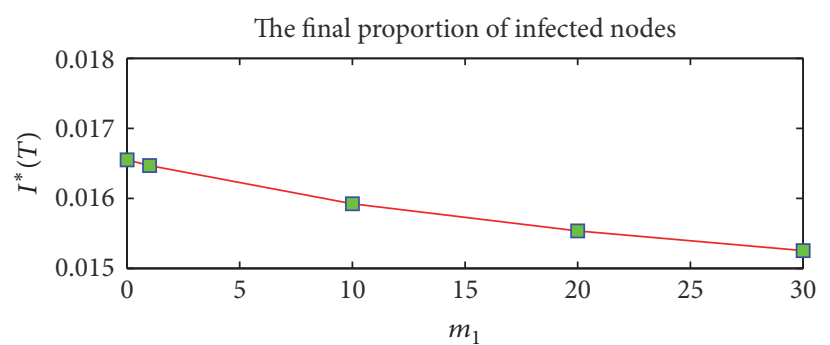

$\neg-I^{*}(T)$

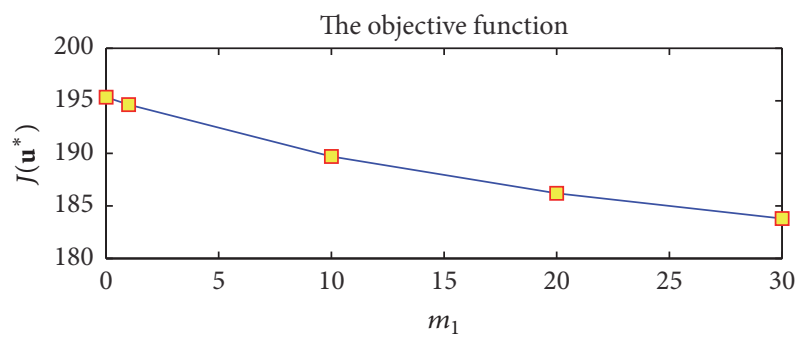

$\neg-J\left(\mathbf{u}^{*}\right)$

(a) $m_{2}=5$

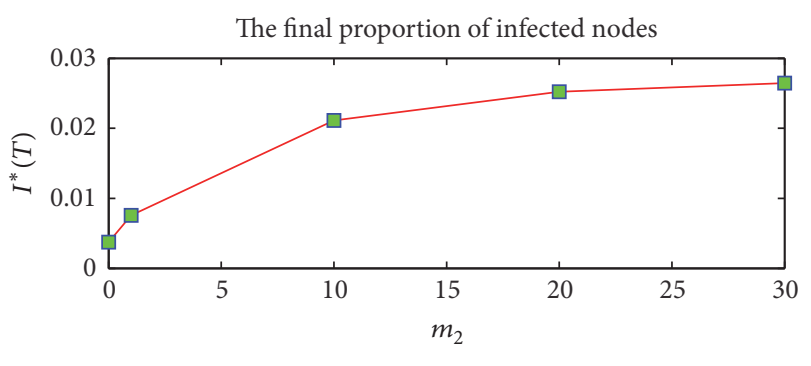

$\neg-I^{*}(T)$

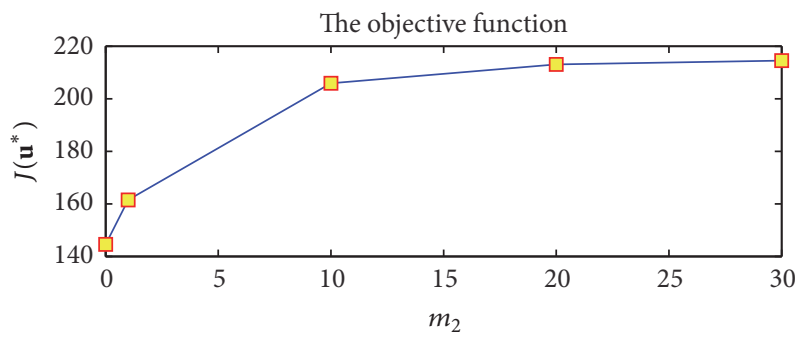

$-\square J\left(\mathbf{u}^{*}\right)$

FIGURE 5: The final proportion of infected nodes $I^{*}(T)$ and the objective function $J\left(\mathbf{u}^{*}\right)$ for different $m_{1}$ and $m_{2}$ for Example 1.

\section{Numerical Examples}

In this section, the effectiveness of the optimal dynamic countermeasure will be verified by some numerical examples.

For our purpose, three networks are considered: a synthetic small-world network (WS network [38]), a synthetic scale-free network (BA network [39]), and a partial Facebook network [40], with $N=150$ nodes, respectively. The parameters of system (33) are set as $\alpha_{i}=0.01, \beta_{i}=0.004887$ (the value of $\beta_{i}$ comes from a report on some real infection probabilities in [41]), $\theta_{i}=0.02, \varepsilon_{i}=1, \gamma=0.01, \bar{\gamma}=0.1$, and $T=50,1 \leq i \leq N$, and the initial conditions are set as $I_{i}(0)=0.03$ and $C_{i}(0)=0.01,1 \leq i \leq N$. The optimality system (33) is solved by invoking the backward-forward Euler 


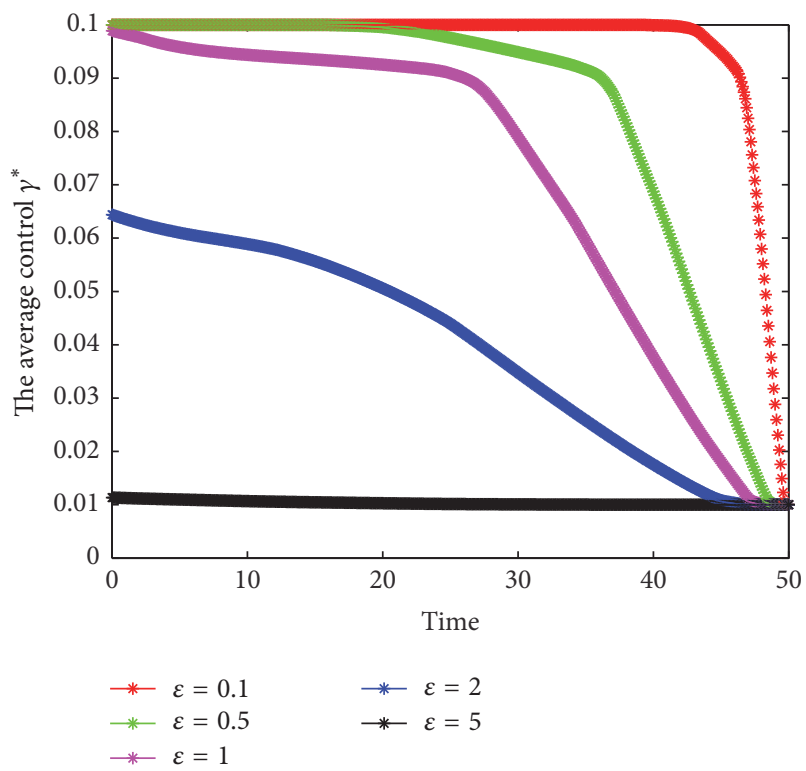

(a) $\gamma^{*}(t)$

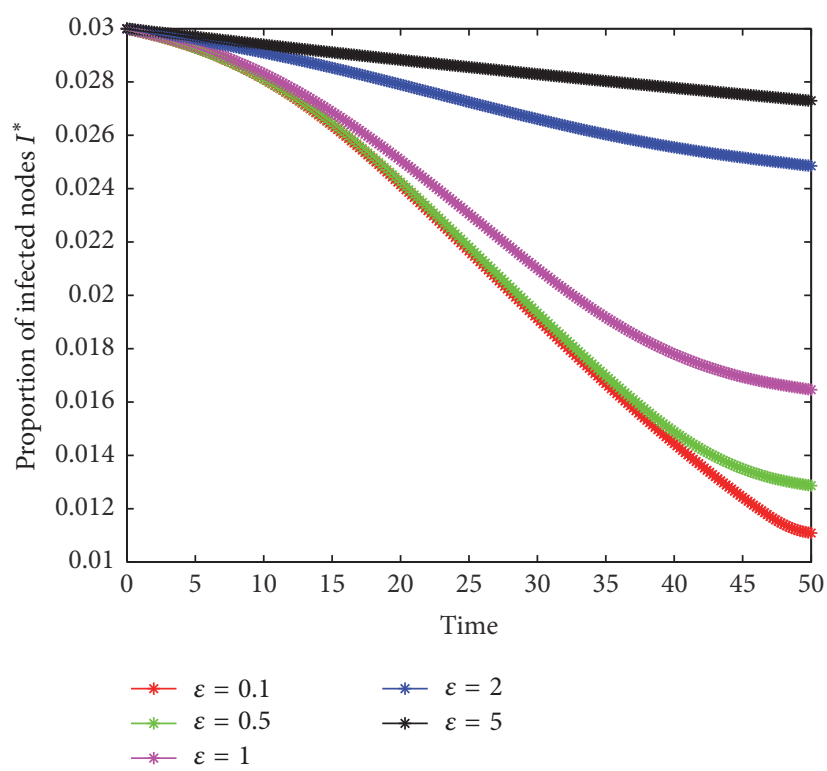

(b) $I^{*}(t)$
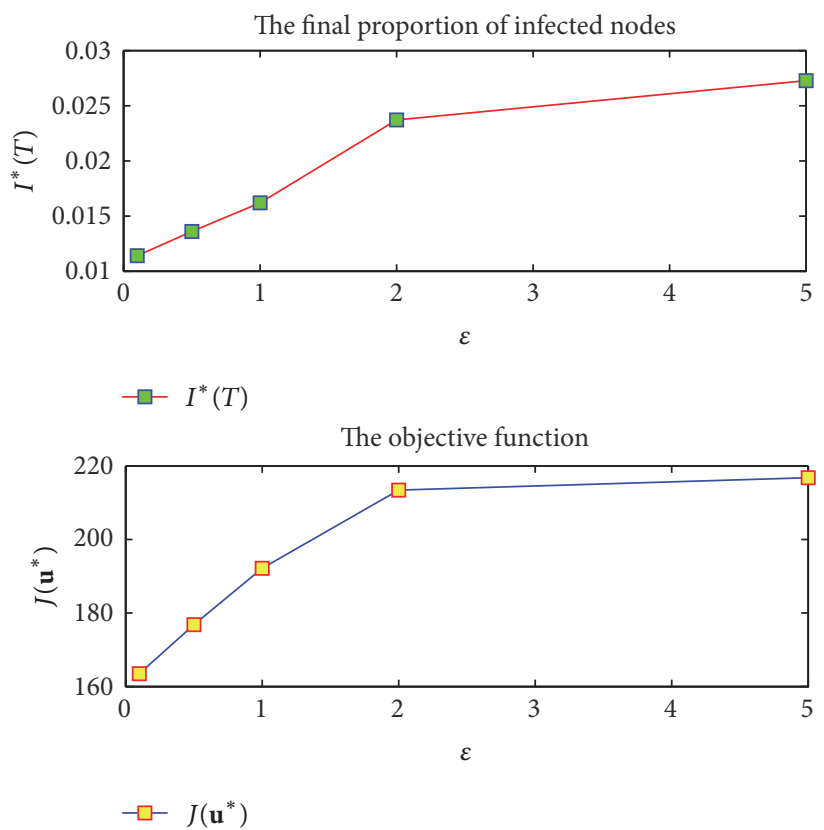

(c) $I^{*}(T)$ and $J\left(\mathbf{u}^{*}\right)$

Figure 6: $\gamma^{*}(t), I^{*}(t), I^{*}(T)$, and $J\left(\mathbf{u}^{*}\right)$ for different $\varepsilon$ with $m_{1}=m_{2}=5$ for Example 1.

scheme with step size 0.01 . Here we have to point out that some parameter values are chosen hypothetically due to the unavailability of real world data.

Suppose $\mathbf{u}^{*}(t)$ is an optimal control for problem (P) and $\mathbf{x}^{*}(t)$ is a solution to the corresponding controlled system. Let $\gamma^{*}(t)$ and $I^{*}(t)$ denote the average control and the proportion of infected nodes under $\mathbf{u}^{*}(t)$, respectively, where

$$
\begin{aligned}
\gamma^{*}(t) & =\frac{1}{N} \sum_{i} \gamma_{i}^{*}(t), \\
I^{*}(t) & =\frac{1}{N} \sum_{i} I_{i}^{*}(t) .
\end{aligned}
$$

Example 1. Take a WS network with 150 nodes and 150 links as the propagation network.

Figure 2 exhibits the average control $\gamma^{*}(t)$ and $I^{*}(t)$ under different control strategies. Table 1 gives the final proportion of infected nodes and the value of objective function $J$ under different control strategies, where the value of static control $\mathbf{u}=0.08895$ is an average of several real curing probabilities reported in [42]. From Figure 2 and Table 1, one can conclude that $\mathbf{u}^{*}$ is indeed the optimal control strategy to minimize the objective function $J$ and reduce virus prevalence to a low level simultaneously. 


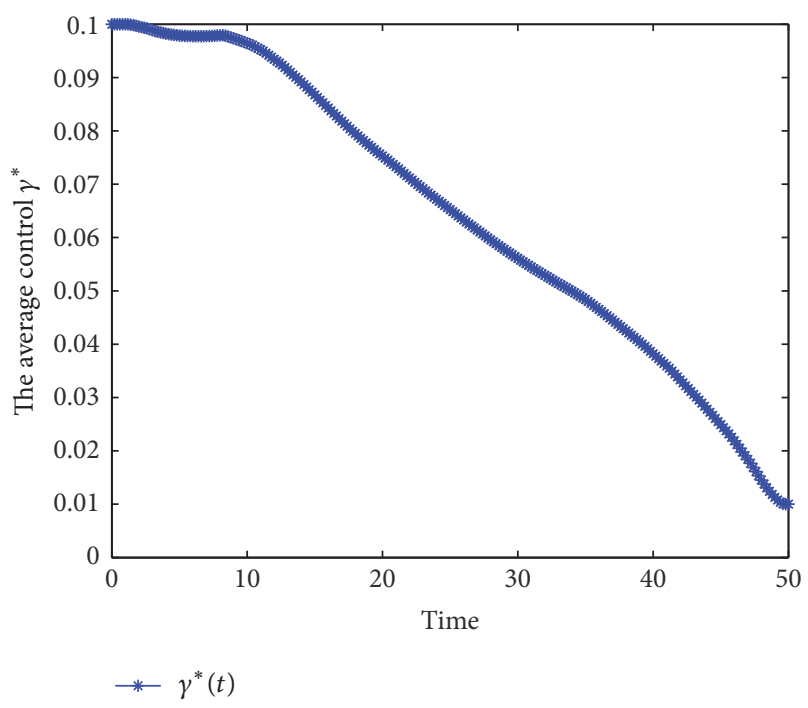

(a) $\gamma^{*}(t)$

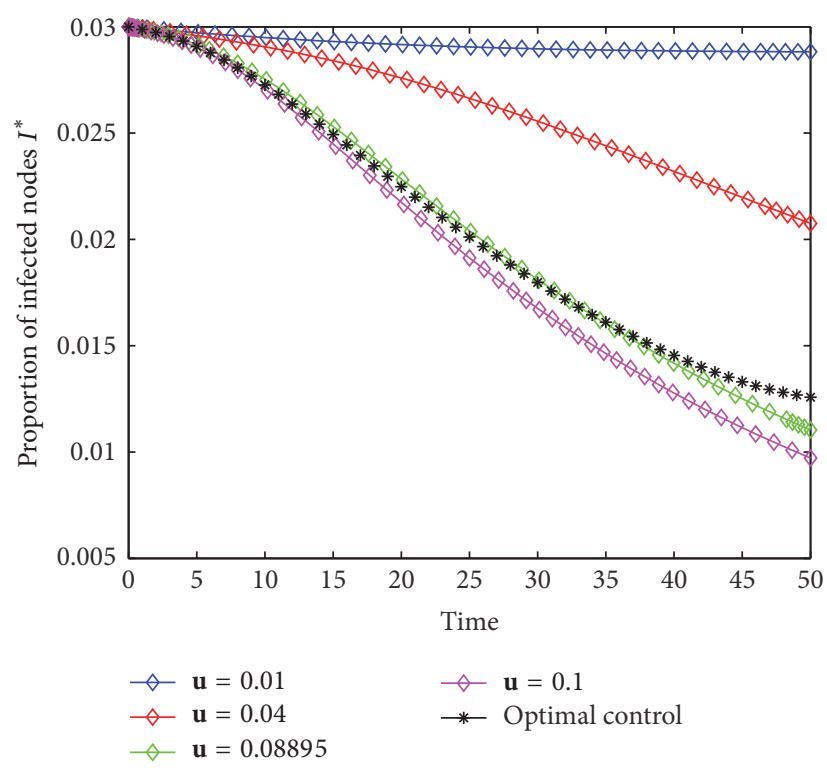

(b) $I^{*}(t)$

FIGURE 7: $\gamma^{*}(t)$ and $I^{*}(t)$ under different control strategies with $m_{1}=m_{2}=2$ for Example 2 .

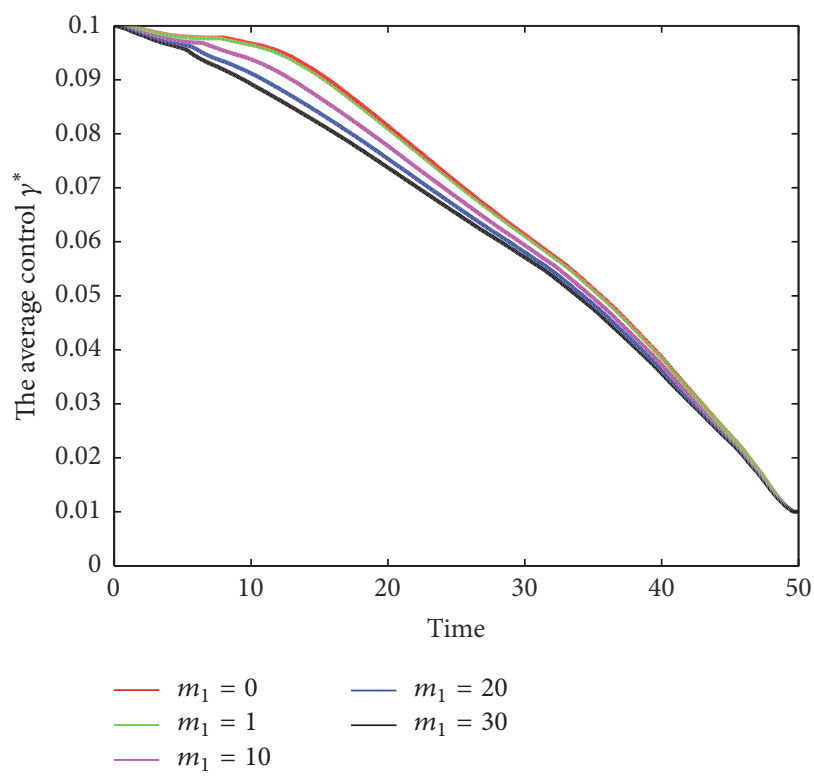

(a) $m_{2}=5$

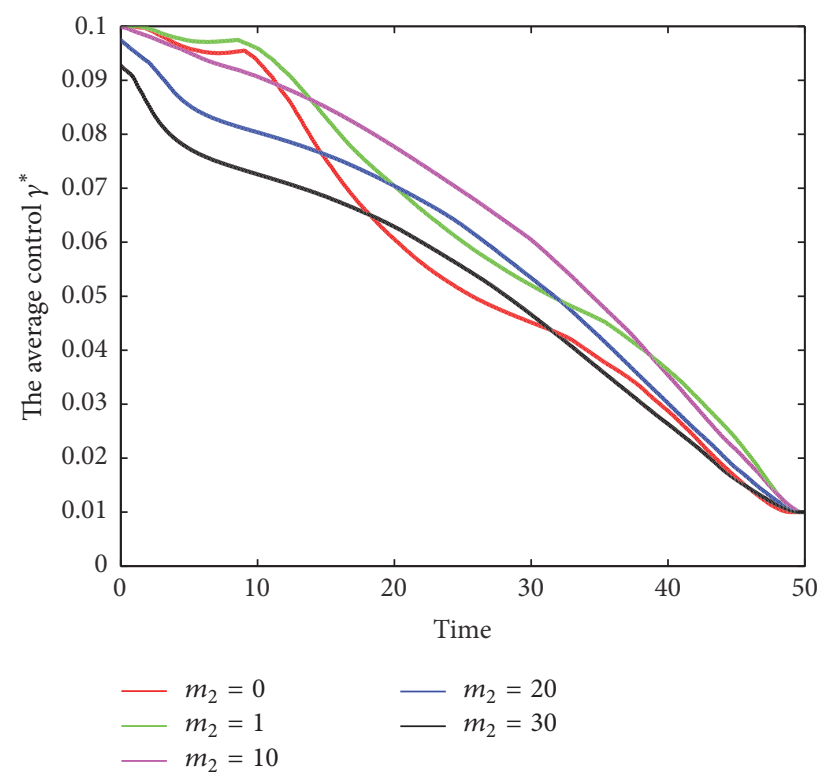

(b) $m_{1}=5$

FIgURE 8: $\gamma^{*}(t)$ for different $m_{1}$ and $m_{2}$ for Example 2.

TABLE 1: $I^{*}(T)$ and $J$ under different control strategies with $m_{1}=m_{2}=2$ for Example 1 .

\begin{tabular}{llcccc}
\hline & $\mathbf{u}=\mathbf{u}^{*}$ & $\mathbf{u}=0.01$ & $\mathbf{u}=0.04$ & $\mathbf{u}=0.08995$ & $\mathbf{u}=0.1$ \\
\hline$I^{*}(T)$ & 0.0089 & 0.0283 & 0.0231 & 0.0072 \\
$J(\mathbf{u})$ & 172.55 & 218.97 & 211.61 & 181.14 & 0.0053 \\
\hline
\end{tabular}

Figure 3 demonstrates the average control $\gamma^{*}(t)$ for different $m_{1}$ and $m_{2}$. From this figure, one can see that (a) enhancing $m_{1}$ and $m_{2}$ roughly reduces $\gamma^{*}(t)$, (b) the smaller $m_{2}$ is, the longer $\gamma^{*}(t)$ stays at $\bar{\gamma}$, and (c) $m_{2}$ has a more significant impact on $\gamma^{*}(t)$ than $m_{1}$ does.
Figure 4 displays $I^{*}(t)$ for different $m_{1}$ and $m_{2}$. From this figure, it can be seen that (a) lower $m_{1}$ favors virus spreading, whereas lower $m_{2}$ is conducive to the containment of virus prevalence, (b) $m_{2}$ affects $I^{*}(t)$ more significantly than $m_{1}$ does, which implies that dynamic countermeasure plays a 


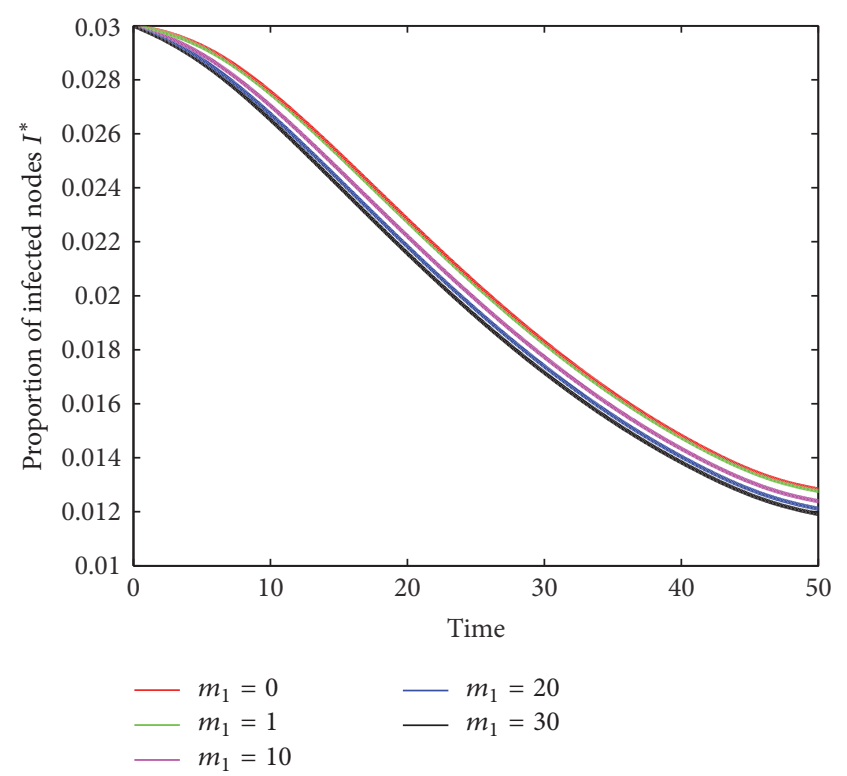

(a) $m_{2}=5$

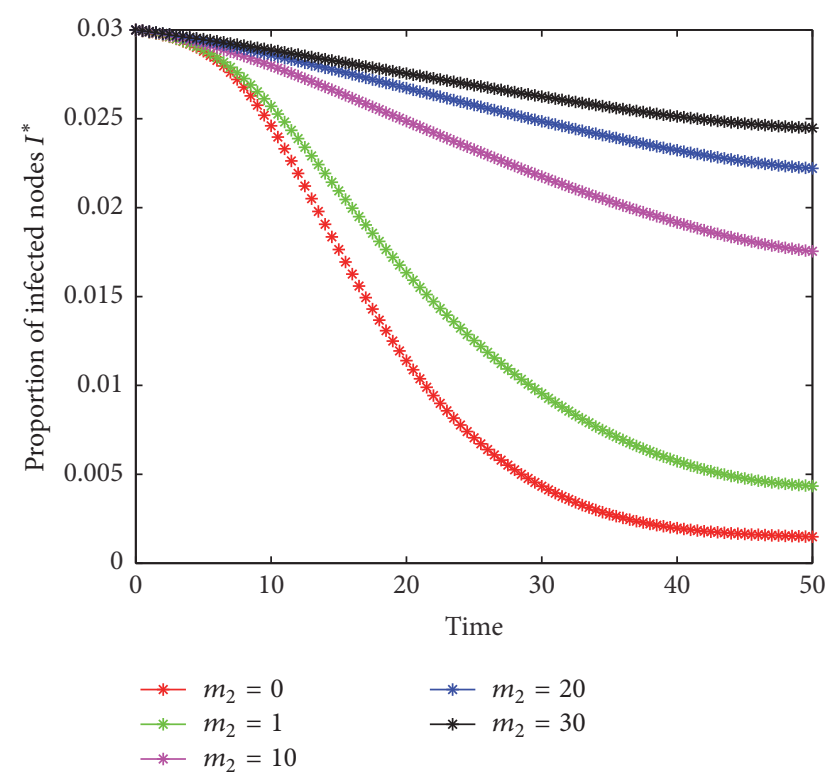

(b) $m_{1}=5$

FIgURE 9: $I^{*}(t)$ for different $m_{1}$ and $m_{2}$ for Example 2.
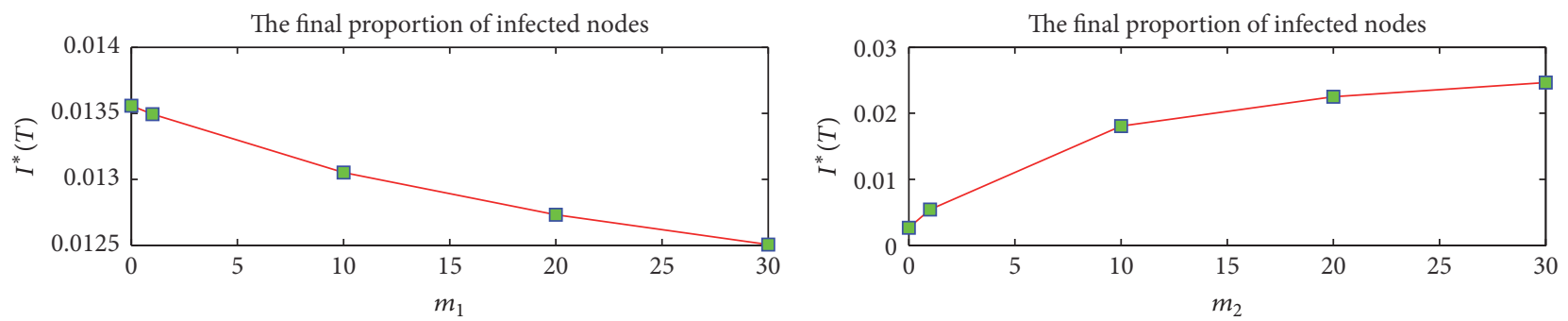

$\neg-I^{*}(T)$

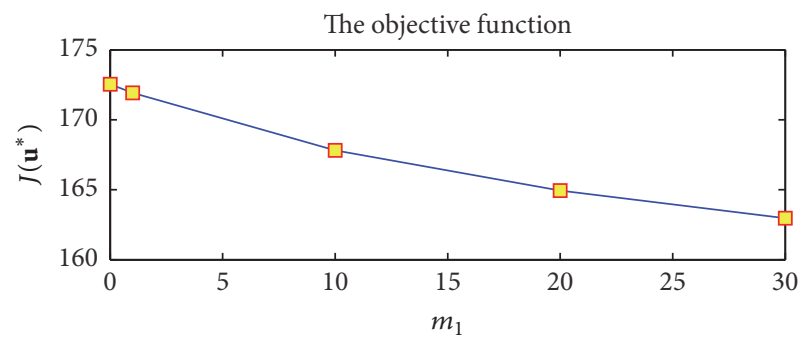

$-\square-J\left(\mathbf{u}^{*}\right)$

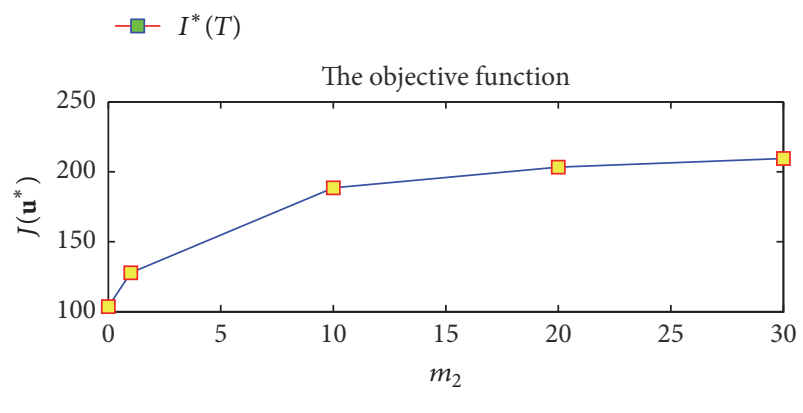

$-\square-J\left(\mathbf{u}^{*}\right)$

(a) $m_{2}=5$

(b) $m_{1}=5$

Figure 10: $I^{*}(T)$ and $J\left(\mathbf{u}^{*}\right)$ for different $m_{1}$ and $m_{2}$ for Example 2.

dominant role in the suppression of virus diffusion, and (c) linear infection rate overestimates virus prevalence, which is in accordance with the result in [7].

Figure 5 depicts the final proportion of infected nodes $I^{*}(T)$ and the objective function $J\left(\mathbf{u}^{*}\right)$ for varied $m_{1}$ and $m_{2}$. From this figure, it can be seen that $J$ is decreasing and increasing with respect to $m_{1}$ and $m_{2}$, respectively, which makes a suggestion that enhancing $m_{1}$ and diminishing $m_{2}$ are beneficial to the containment of viral spread and reduce $J$ to a low level simultaneously.

Figure 6 shows $\gamma^{*}(t), I^{*}(t), I^{*}(T)$, and $J\left(\mathbf{u}^{*}\right)$ for different $\varepsilon$. From this figure, it is found that decreasing $\varepsilon$ is effective on the suppression of virus propagation and attains a lower $J\left(\mathbf{u}^{*}\right)$ simultaneously, although it creates more control cost. This is in good agreement with the fact that when the control effect (i.e., to obtain a low level of infections) is given priority 


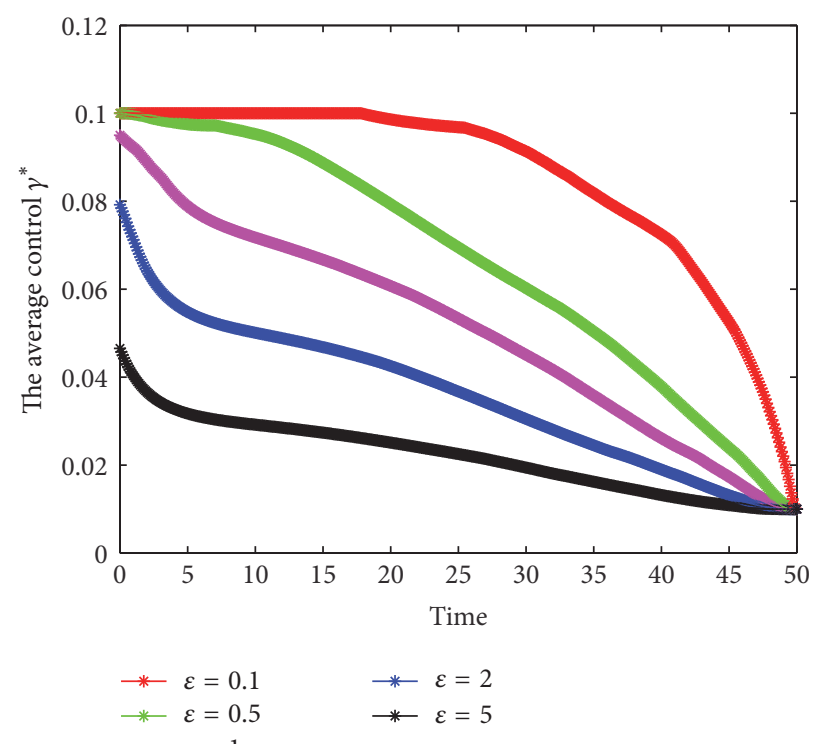

(a) $\gamma^{*}(t)$

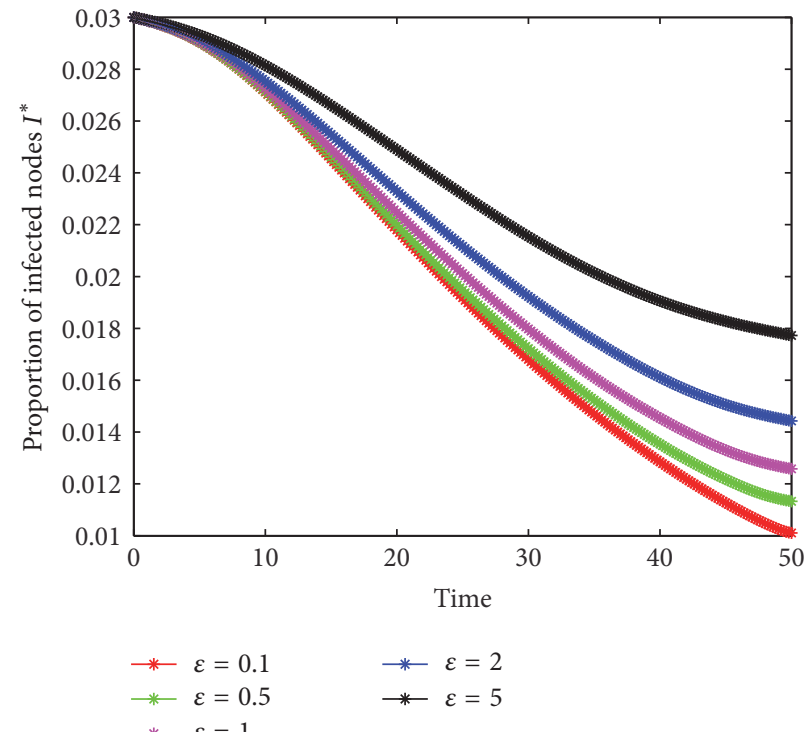

(b) $I^{*}(t)$
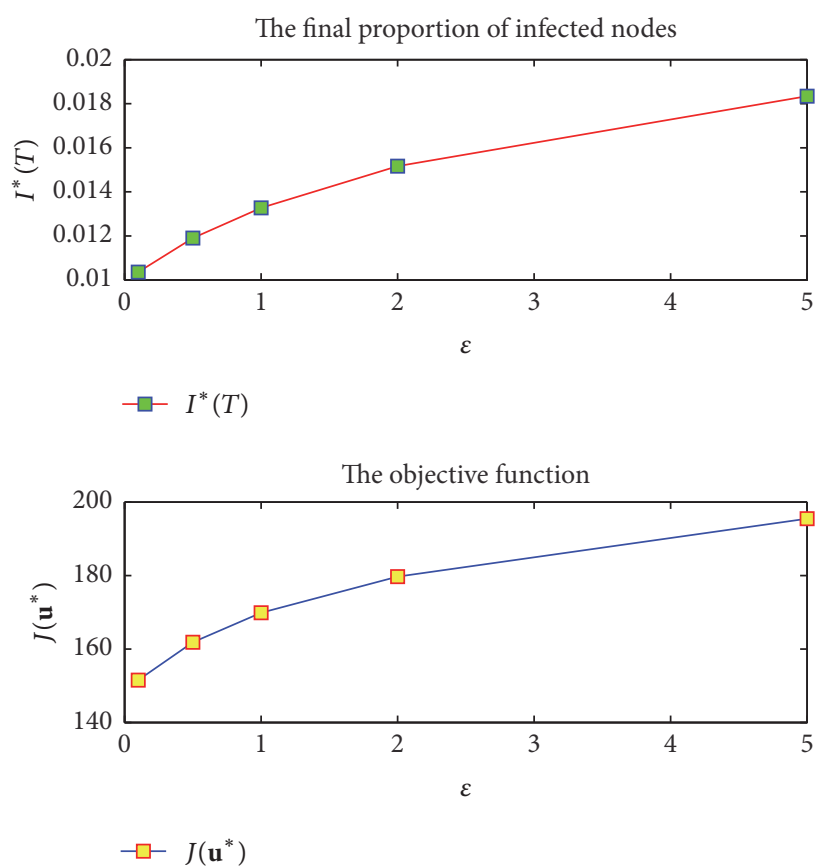

(c) $I^{*}(T)$ and $J\left(\mathbf{u}^{*}\right)$

Figure 11: $\gamma^{*}(t), I^{*}(t), I^{*}(T)$, and $J\left(\mathbf{u}^{*}\right)$ for different $\varepsilon$ with $m_{1}=m_{2}=5$ for Example 2.

(i.e., with lower $\varepsilon$ ), often the decision is made to spend enough control cost. Hence, the tradeoff factor $\varepsilon$ plays a critical role in the balance between control effect and control cost.

Example 2. Take a BA network with 150 nodes and 150 links as the propagation network.

Figure 7 displays $\gamma^{*}(t)$ and $I^{*}(t)$ under different control strategies. Table 2 shows the values of $I^{*}(T)$ and $J(\mathbf{u})$ under different control strategies. Figures 8 and 9 depict $\gamma^{*}(t)$ and $I^{*}(t)$ for different $m_{1}$ and $m_{2}$, respectively. Figure 10 demonstrates $I^{*}(T)$ and $J\left(\mathbf{u}^{*}\right)$ for different $m_{1}$ and $m_{2}$. Figure 11 exhibits $\gamma^{*}(t), I^{*}(t), I^{*}(T)$, and $J\left(\mathbf{u}^{*}\right)$ for different $\varepsilon$. From them, one can get the same results in Example 1. So they are omitted here for brevity.

Example 3. Take a partial Facebook network with 150 nodes and 603 links as the propagation network.

Figure 12 shows $\gamma^{*}(t)$ and $I^{*}(t)$ under different control strategies. Table 3 gives the values of $I^{*}(T)$ and $J(\mathbf{u})$ under different control strategies. Figures 13 and 14 display $\gamma^{*}(t)$ 


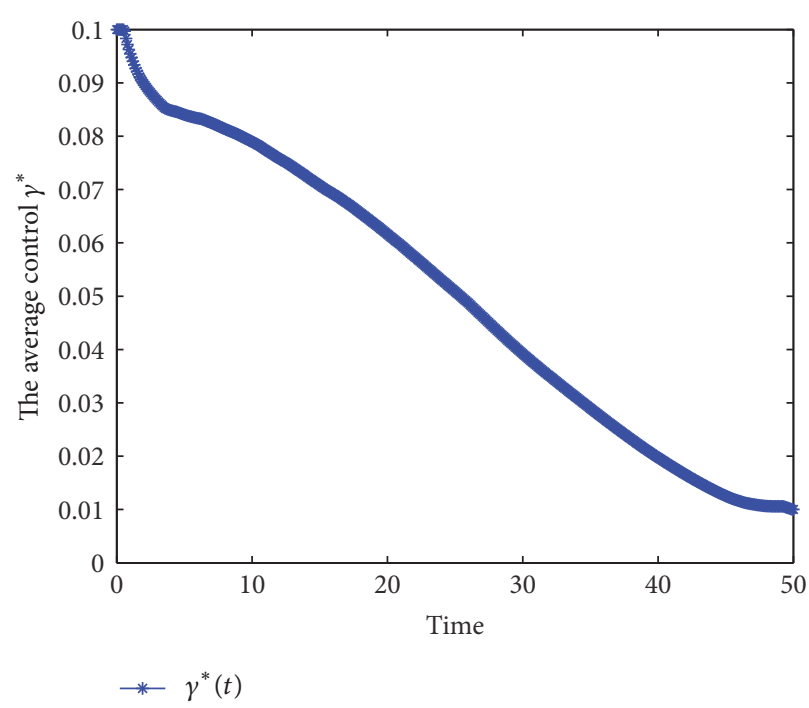

(a) $\gamma^{*}(t)$
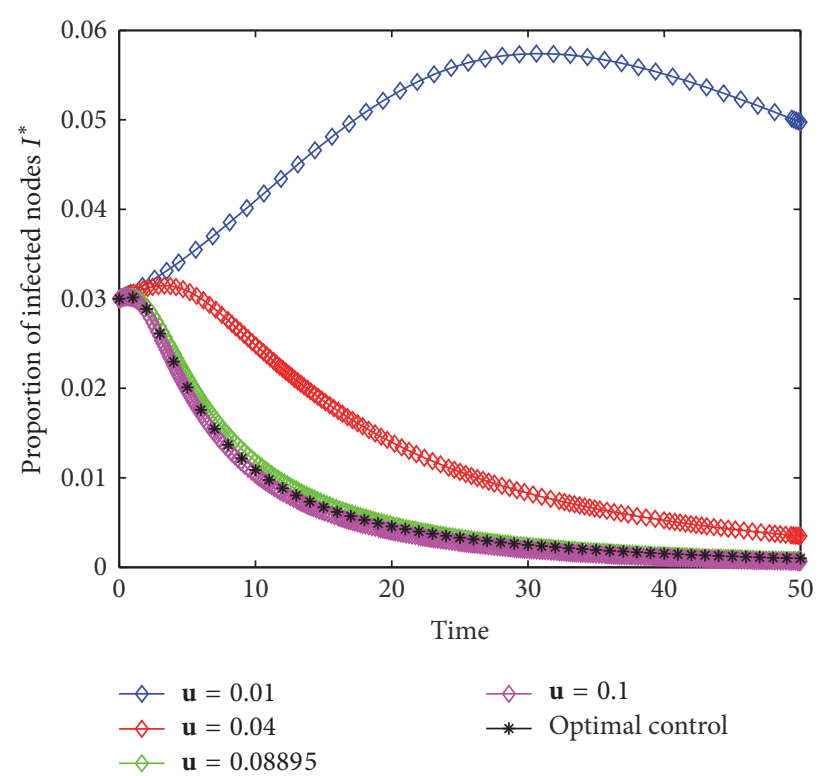

(b) $I^{*}(t)$

FIGURE 12: $\gamma^{*}(t)$ and $I^{*}(t)$ under different control strategies with $m_{1}=m_{2}=2$ for Example 3 .

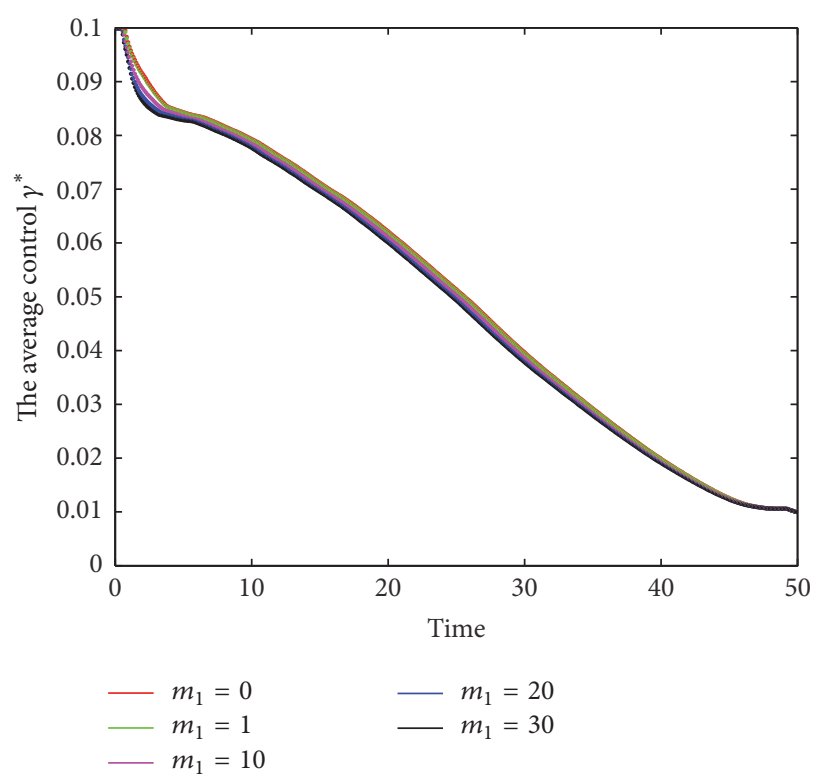

(a) $m_{2}=5$

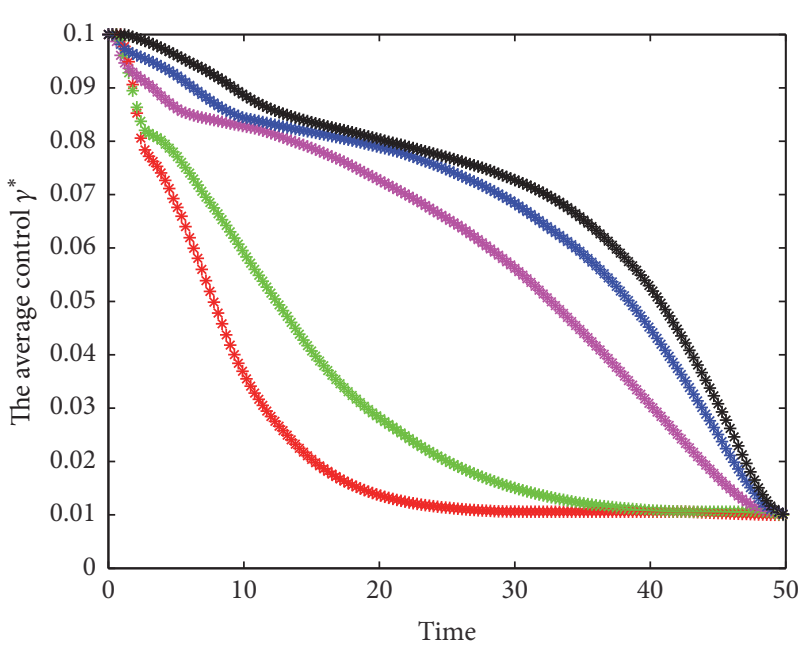

$$
\begin{aligned}
& \text { * } m_{2}=0 \\
& \text { * } m_{2}=1 \\
& \text { * } m_{2}=10 \\
& \text { * } m_{2}=20 \\
& \rightarrow m_{2}=30
\end{aligned}
$$

(b) $m_{1}=5$

FIGURE 13: $\gamma^{*}(t)$ for different $m_{1}$ and $m_{2}$ for Example 3.

TABLE 2: $I^{*}(T)$ and $J$ under different control strategies with $m_{1}=m_{2}=2$ for Example 2.

\begin{tabular}{lccccc}
\hline & $\mathbf{u}=\mathbf{u}^{*}$ & $\mathbf{u}=0.01$ & $\mathbf{u}=0.04$ & $\mathbf{u}=0.08895$ & $\mathbf{u}=0.1$ \\
\hline$I^{*}(T)$ & 0.0132 & 0.0288 & 0.0208 & 0.0111 & 0.0097 \\
$J(\mathbf{u})$ & 169.84 & 219.58 & 202.91 & 185.27 & 185.65 \\
\hline
\end{tabular}

TABLE 3: $I^{*}(T)$ and $J$ under different control strategies with $m_{1}=m_{2}=2$ for Example 3 .

\begin{tabular}{lccccc}
\hline & $\mathbf{u}=\mathbf{u}^{*}$ & $\mathbf{u}=0.01$ & $\mathbf{u}=0.04$ & $\mathbf{u}=0.08895$ & $\mathbf{u}=0.1$ \\
\hline$I^{*}(T)$ & 0.0013 & 0.0504 & 0.0035 & 0.0008 & 0.0006 \\
$J(\mathbf{u})$ & 61.91 & 373.77 & 112.31 & 83.53 & 86.17 \\
\hline
\end{tabular}




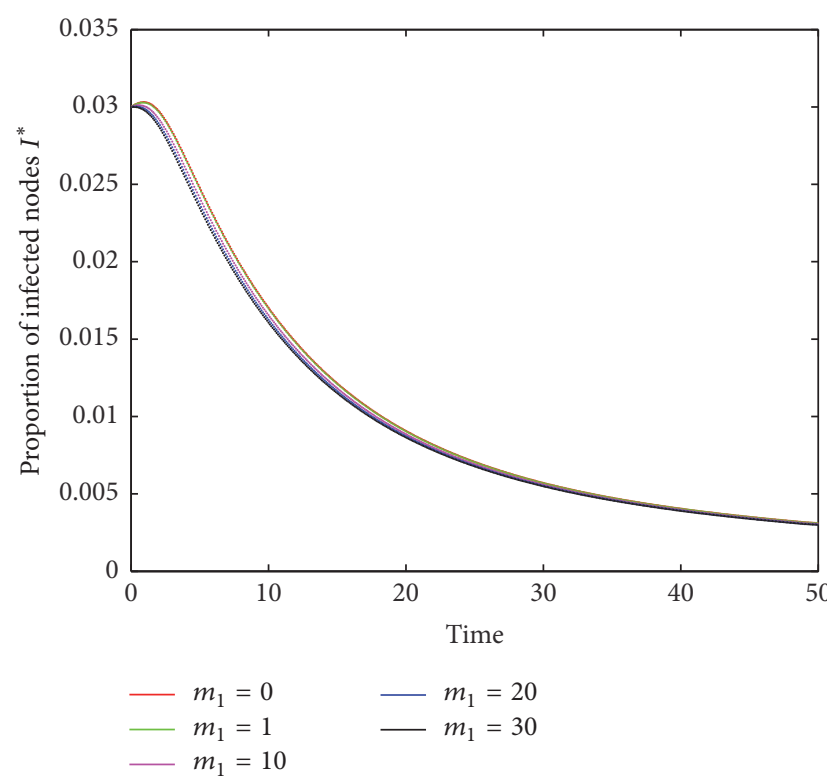

(a) $m_{2}=5$

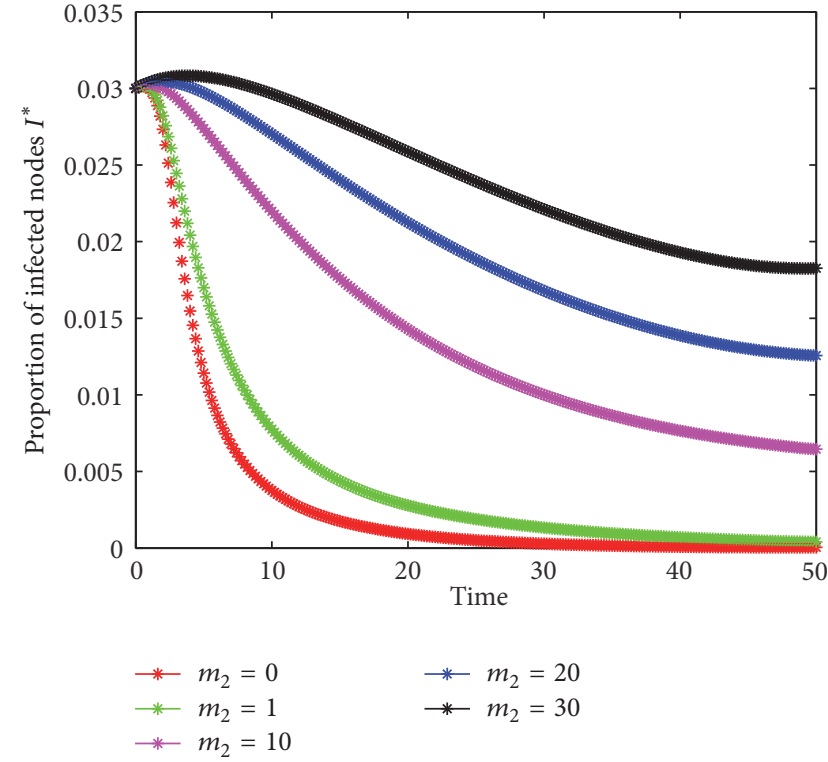

(b) $m_{1}=5$

FiguRe 14: $I^{*}(t)$ for different $m_{1}$ and $m_{2}$ for Example 3.
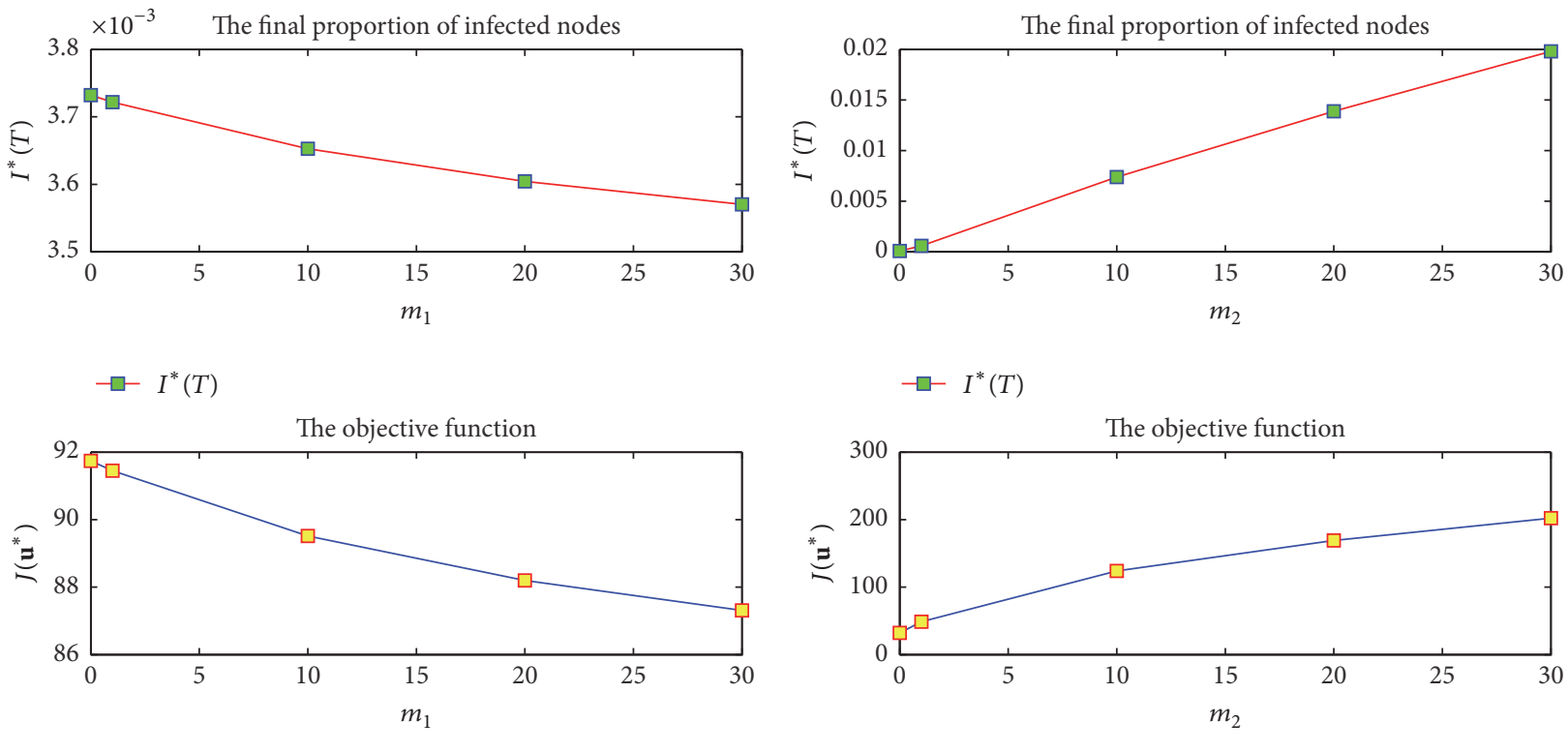

$\neg-J\left(\mathbf{u}^{*}\right)$

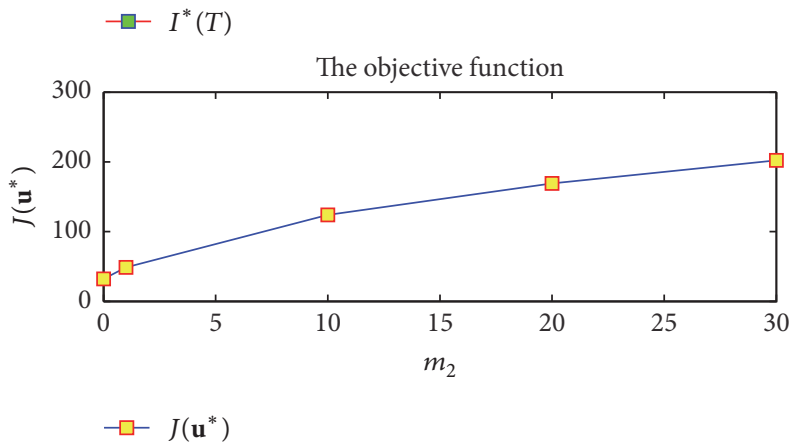

(a) $m_{2}=5$

(b) $m_{1}=5$

Figure 15: $I^{*}(T)$ and $J\left(\mathbf{u}^{*}\right)$ for different $m_{1}$ and $m_{2}$ for Example 3.

and $I^{*}(t)$ for different $m_{1}$ and $m_{2}$, respectively. Figure 15 demonstrates $I^{*}(T)$ and $J\left(\mathbf{u}^{*}\right)$ for varied $m_{1}$ and $m_{2}$. Figure 16 depicts $\gamma^{*}(t), I^{*}(t), I^{*}(T)$, and $J\left(\mathbf{u}^{*}\right)$ for different $\varepsilon$.

Most of the results concluded from this example are the same as those in Example 1 except the two phenomena listed as follows: (a) higher $m_{2}$ increases $\gamma^{*}(t)$, which is contrary to the results in Figures 3(b) and 8(b), and (b) $m_{1}$ has a negligible impact on $\gamma^{*}(t)$ and $I^{*}(t)$. This indicates that the network structure, to some extent, determines the control cost and virus diffusion.

Combining the above numerical examples, the main results are listed below.

(a) $\mathbf{u}^{*}$ is indeed the optimal control strategy to minimize the objective function $J$ and reduce the infections to a low level simultaneously. 


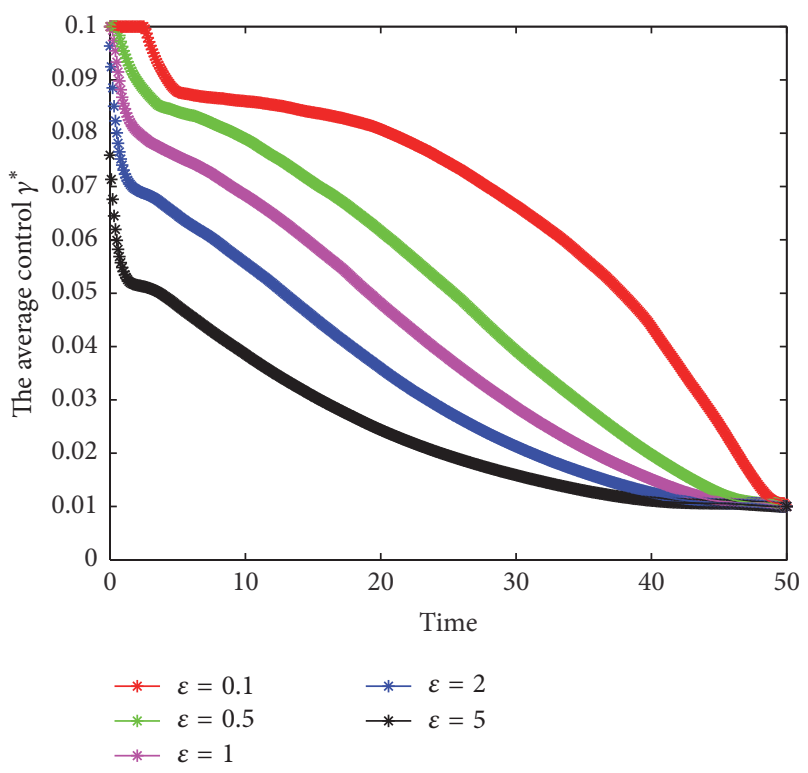

(a) $\gamma^{*}(t)$

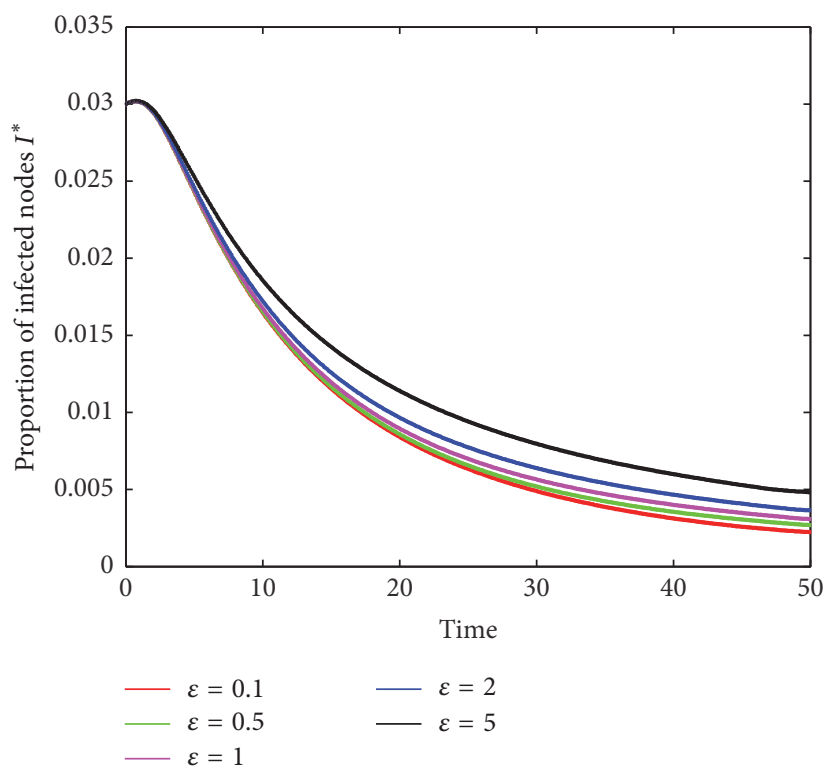

(b) $I^{*}(t)$
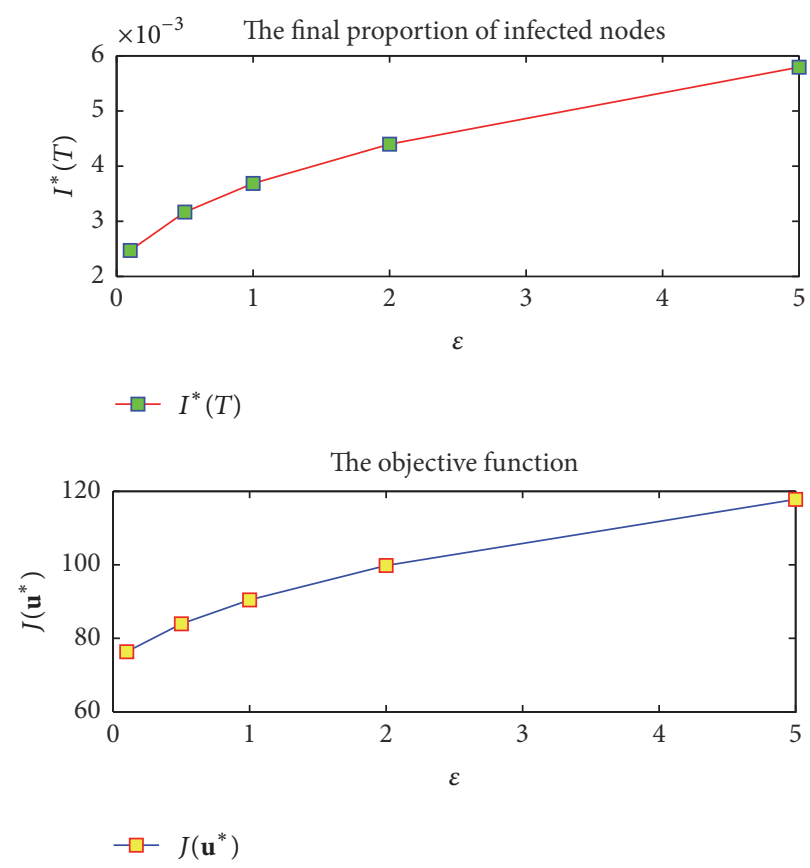

(c) $I^{*}(T)$ and $J\left(\mathbf{u}^{*}\right)$

Figure 16: $\gamma^{*}(t), I^{*}(t), I^{*}(T)$, and $J\left(\mathbf{u}^{*}\right)$ for different $\varepsilon$ with $m_{1}=m_{2}=5$ for Example 3 .

(b) Linear infection rate overestimates the prevalence of virus.

(c) Enhancing $m_{1}$ and diminishing $m_{2}$ are conductive to the containment of viral propagation and reduce $J$ to a low level simultaneously.

(d) $m_{2}$ has more significant influences on $\gamma^{*}(t), I^{*}(t)$, and $J\left(\mathbf{u}^{*}\right)$ than $m_{1}$ does.

(e) Decreasing the tradeoff factor $\varepsilon$ is beneficial to the suppression of virus spread and obtains a lower $J\left(\mathbf{u}^{*}\right)$ simultaneously, although it brings more control cost.
Additionally, the structure of network, to some extent, determines the virus prevalence and the control cost. Thus, we shall investigate how the network topology affects virus spreading and control cost in the next work.

\section{Concluding Remarks}

This paper has studied the issue of how to work out an optimal dynamic countermeasure for achieving a low level of infections with a low cost. In this regard, a controlled node-level SICS model with nonlinear infection rate has 
been established. Furthermore, an optimal control problem has been proposed. The existence of an optimal control and the corresponding optimality system have also been derived. Additionally, some numerical examples have been given to illustrate the main results. Specifically, it has been found that the proposed optimal countermeasure scheme can achieve a low level of infections at a low cost.

In our opinions, the next work could be made as follows. First, the quadratic cost functions may be generalized to some generic functions. Second, delays [43-45], pulses [46, 47], and random fluctuations [15] may be incorporated to controlled node-level models. Last, but not least, it is worthy to carry out research on the impact of the network topology $[9,25,48,49]$ on the dynamic countermeasure strategy.

\section{Conflicts of Interest}

The authors declare that there are no conflicts of interest regarding the publication of this paper.

\section{Acknowledgments}

This work is supported by Natural Science Foundation of China (Grant nos. 61572006 and 61503307), Science and Technology Research Program of Chongqing Municipal Education Commission (Grant nos. KJ1500415, KJ1704080, and KJ1704081), and Doctoral Scientific Research Foundation of Chongqing University of Posts and Telecommunications (Grant nos. A2015-02 and A2016-10).

\section{References}

[1] J. O. Kephart and S. R. White, "Directed-graph epidemiological models of computer viruses," in Proceedings of the IEEE Computer Society Symposium on Research in Security and Privacy, pp. 343-359, Oakland, Calif, USA, May 1991.

[2] J. R. C. Piqueira, A. A. de Vasconcelos, C. E. C. J. Gabriel, and V. O. Araujo, "Dynamic models for computer viruses," Computers and Security, vol. 27, no. 7-8, pp. 355-359, 2008.

[3] B. K. Mishra and S. K. Pandey, "Dynamic model of worms with vertical transmission in computer network," Applied Mathematics and Computation, vol. 217, no. 21, pp. 8438-8446, 2011.

[4] C. Gan, X. Yang, W. Liu, Q. Zhu, and X. Zhang, "Propagation of computer virus under human intervention: a dynamical model," Discrete Dynamics in Nature and Society, vol. 2012, Article ID 106950, 8 pages, 2012.

[5] L.-X. Yang, X. Yang, L. Wen, and J. Liu, "A novel computer virus propagation model and its dynamics," International Journal of Computer Mathematics, vol. 89, no. 17, pp. 2307-2314, 2012.

[6] Q. Zhu, X. Yang, L.-X. Yang, and X. Zhang, "A mixing propagation model of computer viruses and countermeasures," Nonlinear Dynamics, vol. 73, no. 3, pp. 1433-1441, 2013.

[7] P. Van Mieghem, J. Omic, and R. Kooij, "Virus spread in networks," IEEE/ACM Transactions on Networking, vol. 17, no. 1, pp. 1-14, 2009.

[8] F. D. Sahneh and C. M. Scoglio, "Optimal information dissemination in epidemic networks," in Proceedings of the 51st IEEE Conference on Decision and Control (CDC '12), pp. 1657-1662, December 2012.
[9] L.-X. Yang, M. Draief, and X. Yang, "The impact of the network topology on the viral prevalence: a node-based approach," PLoS ONE, vol. 10, no. 7, article e0134507, 2015.

[10] L.-X. Yang, M. Draief, and X. Yang, "The optimal dynamic immunization under a controlled heterogeneous node-based SIRS model," Physica A, vol. 450, pp. 403-415, 2016.

[11] C. Gan, "Modeling and analysis of the effect of network eigenvalue on viral spread," Nonlinear Dynamics, vol. 84, no. 3, pp. 1727-1733, 2016.

[12] F. Cohen, "Computer viruses: theory and experiments," Computers \& Security, vol. 6, no. 1, pp. 22-35, 1987.

[13] W. H. Murray, "The application of epidemiology to computer viruses," Computers and Security, vol. 7, no. 2, pp. 139-145, 1988.

[14] J. R. Piqueira and V. O. Araujo, "A modified epidemiological model for computer viruses," Applied Mathematics and Computation, vol. 213, no. 2, pp. 355-360, 2009.

[15] C. Zhang, Y. Zhao, Y. Wu, and S. Deng, "A stochastic dynamic model of computer viruses," Discrete Dynamics in Nature and Society, vol. 2012, Article ID 264874, 16 pages, 2012.

[16] J. Amador and J. R. Artalejo, "Stochastic modeling of computer virus spreading with warning signals," Journal of the Franklin Institute, vol. 350, no. 5, pp. 1112-1138, 2013.

[17] Y. Yao, X. Feng, W. Yang, W. Xiang, and F. Gao, "Analysis of a delayed Internet worm propagation model with impulsive quarantine strategy," Mathematical Problems in Engineering, Article ID 369360, 18 pages, 2014.

[18] C. Gan, X. Yang, W. Liu, and Q. Zhu, "A propagation model of computer virus with nonlinear vaccination probability," Communications in Nonlinear Science and Numerical Simulation, vol. 19, no. 1, pp. 92-100, 2014.

[19] L.-X. Yang and X. Yang, "A new epidemic model of computer viruses," Communications in Nonlinear Science and Numerical Simulation, vol. 19, no. 6, pp. 1935-1944, 2014.

[20] J. Ren, Y. Xu, and J. Liu, "Investigation of dynamics of a virusantivirus model in complex network," Physica A, vol. 421, pp. 533-540, 2015.

[21] B. K. Mishra, K. Haldar, and D. N. Sinha, "Impact of information based classification on network epidemics," Scientific Reports, vol. 6, article 28289, 2016.

[22] J. Ren, J. Liu, and Y. Xu, "Modeling the dynamics of a networkbased model of virus attacks on targeted resources," Communications in Nonlinear Science and Numerical Simulation, vol. 31, no. 1-3, pp. 1-10, 2016.

[23] L. C. Chen and K. M. Carley, "The impact of countermeasure propagation on the prevalence of computer viruses," IEEE Transactions on Systems, Man, and Cybernetics B Cybernetics, vol. 34, no. 2, pp. 823-833, 2004.

[24] Y. Wang, D. Chakrabarti, C. Wang, and C. Faloutsos, "Epidemic spreading in real networks: an eigenvalue viewpoint," in Proceedings of the 22nd International Symposium on Reliable Distributed Systems (SRDS '03), pp. 25-34, Florence, Italy, October 2003.

[25] M. Youssef and C. Scoglio, "An individual-based approach to SIR epidemics in contact networks," Journal of Theoretical Biology, vol. 283, no. 1, pp. 136-144, 2011.

[26] Y. Lin, J. C. S. Lui, K. Jung, and S. Lim, "Modelling multistate diffusion process in complex networks: theory and applications," Journal of Complex Networks, vol. 2, no. 4, pp. 431-459, 2014. 
[27] C. Nowzari, V. M. Preciado, and G. J. Pappas, "Stability analysis of generalized epidemic models over directed networks," in Proceedings of the 2014 53rd IEEE Annual Conference on Decision and Control, CDC 2014, pp. 6197-6202, December 2014.

[28] R. Pastor-Satorras, C. Castellano, P. Van Mieghem, and A. Vespignani, "Epidemic processes in complex networks," Reviews of Modern Physics, vol. 87, no. 3, pp. 925-979, 2015.

[29] Q. Zhu, X. Yang, L.-X. Yang, and C. Zhang, "Optimal control of computer virus under a delayed model," Applied Mathematics and Computation, vol. 218, no. 23, pp. 11613-11619, 2012.

[30] L. Chen, K. Hattaf, and J. Sun, "Optimal control of a delayed SLBS computer virus model," Physica A, vol. 427, pp. 244-250, 2015.

[31] L.-X. Yang, M. Draief, and X. Yang, "The optimal dynamic immunization under a controlled heterogeneous node-based SIRS model," Physica A. Statistical Mechanics and its Applications, vol. 450, pp. 403-415, 2016.

[32] C. Zhang and H. Huang, "Optimal control strategy for a novel computer virus propagation model on scale-free networks," Physica A, vol. 451, pp. 251-265, 2016.

[33] C. Gan, M. Yang, Z. Zhang, and W. Liu, "Global dynamics and optimal control of a viral infection model with generic nonlinear infection rate," Discrete Dynamics in Nature and Society, Article ID 7571017, 9 pages, 2017.

[34] M. I. Kamien and N. L. Schwartz, Dynamic Optimization: The Calculus of Variations and Optimal Control in Economics and Management, Elsevier Science, The Netherlands, 2000.

[35] D. Liberzon, Calculus of Variations and Optimal Control Theory: A Concise Introduction, Princeton University Press, Princeton, NJ, USA, 2012.

[36] R. C. Robinson, An Introduction to Dynamical Systems: Continuous and Discrete, Prentice Hall, New York, NY, USA, 2004.

[37] R. T. Rockafellar, Convex Analysis, Princeton Mathematical Series, No. 28, Princeton University Press, Princeton, NJ, USA, 1970.

[38] D. J. Watts and S. H. Strogatz, "Collective dynamics of "smallworld” networks," Nature, vol. 393, no. 6684, pp. 440-442, 1998.

[39] A.-L. Barabási and R. Albert, "Emergence of scaling in random networks," Science, vol. 286, no. 5439, pp. 509-512, 1999.

[40] http://snap.stanford.edu/data/egonets-Facebook.html.

[41] http://green.wangminjie.cn/newsinfo-136730.html.

[42] http://pcedu.pconline.com.cn/pingce/pingcesystem/1109/ 2527365_all.html.

[43] B. K. Mishra and D. K. Saini, "SEIRS epidemic model with delay for transmission of malicious objects in computer network," Applied Mathematics and Computation, vol. 188, no. 2, pp. 14761482, 2007.

[44] Y. Yao, X.-w. Xie, H. Guo, G. Yu, F.-X. Gao, and X.-j. Tong, "Hopf bifurcation in an Internet worm propagation model with time delay in quarantine," Mathematical and Computer Modelling, vol. 57, no. 11-12, pp. 2635-2646, 2013.

[45] J. Ren and Y. Xu, "Stability and bifurcation of a computer virus propagation model with delay and incomplete antivirus ability," Mathematical Problems in Engineering, Article ID 475934, 9 pages, 2014.

[46] Y. Yao, L. Guo, H. Guo, G. Yu, F. Gao, and X. Tong, "Pulse quarantine strategy of internet worm propagation: modeling and analysis," Computers and Electrical Engineering, vol. 38, no. 5, pp. 1047-1061, 2012.
[47] L.-X. Yang and X. Yang, "The pulse treatment of computer viruses: a modeling study," Nonlinear Dynamics, vol. 76, no. 2, pp. 1379-1393, 2014.

[48] C. Gan, X. Yang, W. Liu, Q. Zhu, J. Jin, and L. He, "Propagation of computer virus both across the Internet and external computers: a complex-network approach," Communications in Nonlinear Science and Numerical Simulation, vol. 19, no. 8, pp. 2785-2792, 2014.

[49] Y. Yang, G. Xie, and J. Xie, "Mining important nodes in directed weighted complex networks," Discrete Dynamics in Nature and Society, vol. 2017, Article ID 9741824, 7 pages, 2017. 


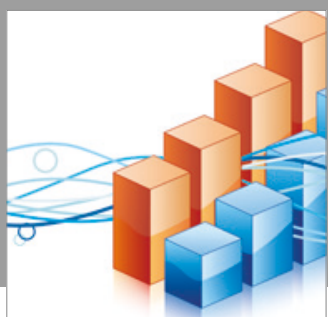

Advances in

Operations Research

vatersals

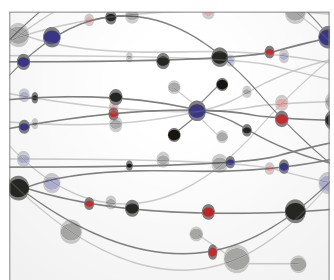

\section{The Scientific} World Journal
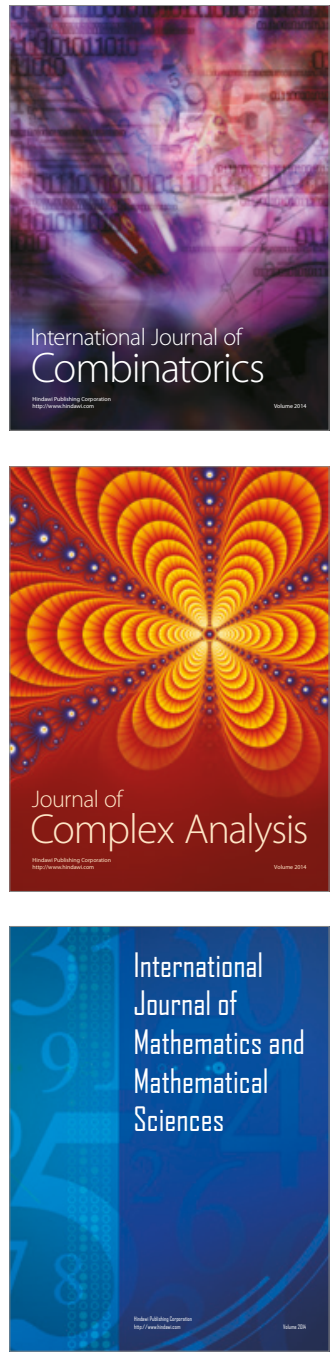
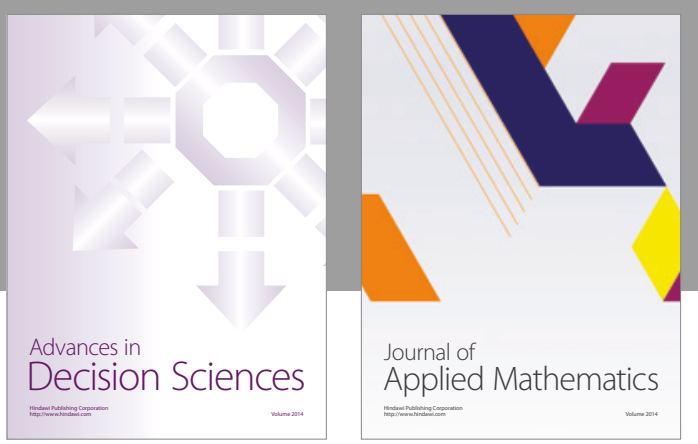

Algebra

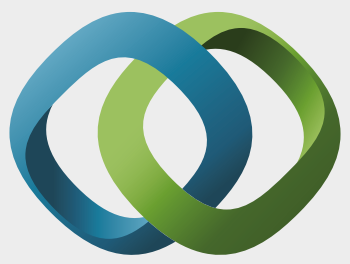

\section{Hindawi}

Submit your manuscripts at

https://www.hindawi.com
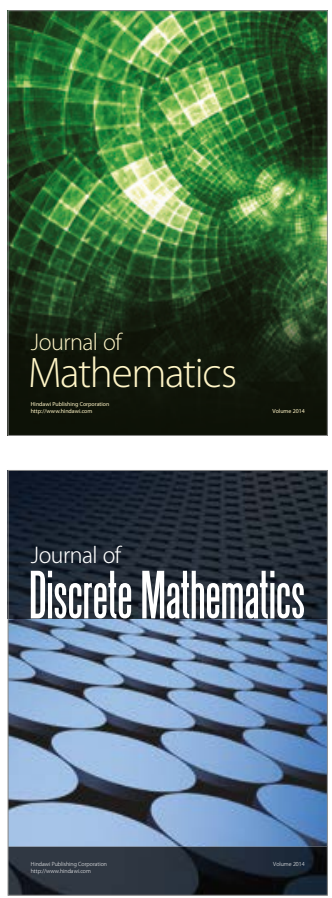

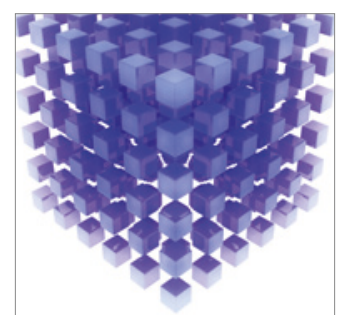

Mathematical Problems in Engineering
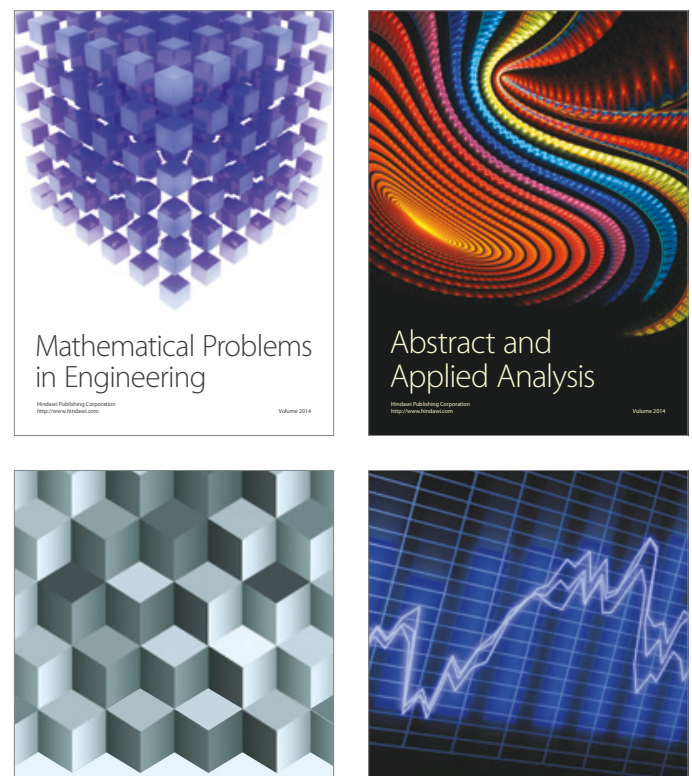

Journal of

Function Spaces

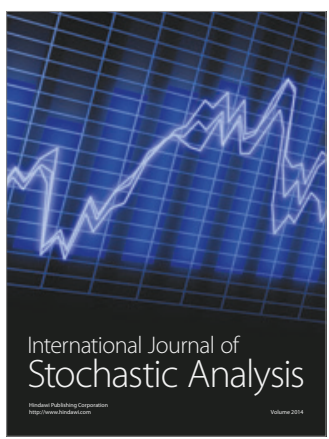

Probability and Statistics
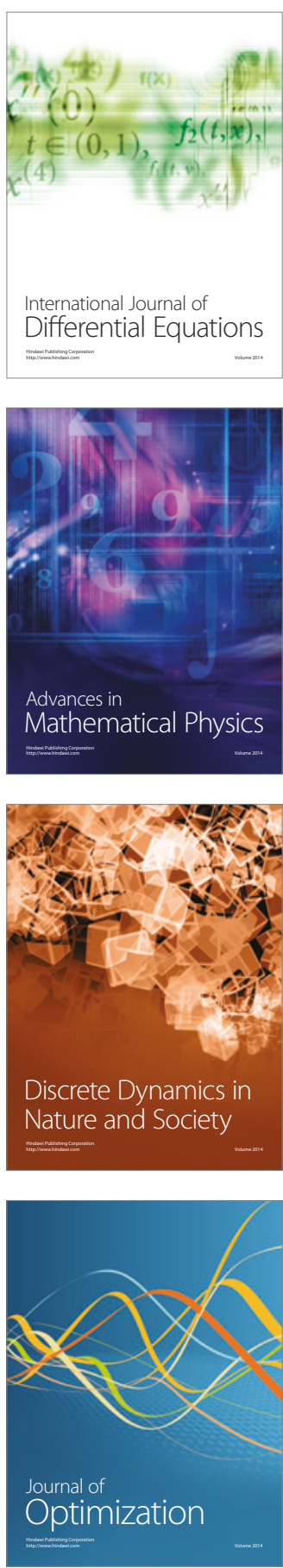